\title{
A new proof of Geroch's theorem on temporal splitting of globally hyperbolic spacetime
}

\author{
Ali Bleybel ( $\square$ bleybel@ul.edu.lb ) \\ Lebanese University https://orcid.org/0000-0001-5946-5476
}

\section{Research Article}

Keywords: Spacetime, Causality, Global hyperbolicity

Posted Date: March 1st, 2021

DOI: https://doi.org/10.21203/rs.3.rs-229207/v1

License: (c) (i) This work is licensed under a Creative Commons Attribution 4.0 International License. Read Full License 


\title{
A NEW PROOF OF GEROCH'S THEOREM ON TEMPORAL SPLITTING OF GLOBALLY HYPERBOLIC SPACETIMES
}

\author{
ALI BLEYBEL
}

\begin{abstract}
In this paper, we use our results concerning temporal foliations of causal sets in order to provide a new proof of Geroch's Theorem on temporal foliations in a globally hyperbolic spacetime.
\end{abstract}

\section{INTRODUCTION}

In this paper, we describe how to recover Geroch's Theorem [6] on the existence of a splitting of a globally hyperbolic spacetime $M$ into Cauchy slices from our results in [3].

The interest of this proof method is twofold. Besides providing a new perspective on the notion of Cauchy hypersurface and its relation with the corresponding notion in the context of causal set theory (keeping in mind that the resemblance is far from perfect), it provides a way to transfer results from the discrete into the continuous settings.

Note that in [2], a similar technique was used in order to show that a (increasing) sequence of nested causets (indexed by $\mathbb{N}$ ) allow to recover the manifold topology. These causets are assumed to be uniformly embedded in the manifold and are generated by a Poisson process, so that, at each stage the density of points of each causet remains constant.

Note that other results in the same direction (i.e. recovering continuum topology from causal sets) were obtained later (see [9], [8]).

After an introductory section, we provide additional results on temporal splitting of causal sets, then we proceed to the proof of the main result (Geroch's Theorem). In section 5 we apply the results already obtained in order to show the existence of a time function on a globally hyperbolic spacetime. In the final section it is shown that the slices have spacelike tangent hyperplanes defined almost everywhere.

Acknowledgement: I wish to thank the reviewers for carefully reading the manuscript, and for providing many helpful comments and corrections.

Key words and phrases. Spacetime, Causality, Global hyperbolicity. 


\section{Notation AND PRELIMINARIES}

In this section I review some background results and notation on ordered sets that will be used throughout the text.

2.1. Causal spaces. A Causal space is a set $\mathcal{X}$ endowed with a partial order relation $\prec$. The point of introducing this terminology is just to emphasize the link with the more restrictive notion of causal sets.

Recall that a partial order $\prec$ is a binary relation that is reflexive $x \prec x$, transitive $x \prec y \& y \prec z \rightarrow x \prec z$ and antisymmetric $(x \prec y \& y \prec x) \rightarrow$ $x=y$ for all $x, y, z \in \mathcal{X}$. Sometimes we write $y \succ x$ to mean $x \prec y$. By $x \supsetneqq y$ we denote $x \prec y \& x \neq y$.

A causal space is dense when $x \prec y, x \neq y$ implies the existence of some $z, z \neq x, z \neq y$ such that $x \prec z \prec y$.

Two elements $x$ and $y$ of a causal space are incomparable if neither $x \prec y$ nor $y \prec x$; this is abbreviated using the notation $x \| y$.

Let $x \in \mathcal{X}$. The set of elements lying above $x$ will be denoted by $x_{\uparrow}$, while those lying below $x$ form the set $x_{\downarrow}$.

Given two elements $x, y \in \mathcal{X}$, we denote by $[x, y]$ (the interval having endpoints $x$ and $y$ respectively) the set

$$
[x, y]:=\{z \in \mathcal{X} \mid x \prec z \prec y\}=x_{\uparrow} \cap y_{\downarrow} .
$$

If $X$ is a subset of $\mathcal{X}$, then:

$$
X_{\downarrow}:=\{y \in \mathcal{X} \mid(\exists x \in X) y \prec x\}, \quad X_{\uparrow}:=\{y \in \mathcal{X} \mid(\exists x \in X) x \prec y\} .
$$

A causal set $\mathcal{X}$ is a locally finite causal space, i.e. the interval $[x, y]$ is finite for all $x, y \in \mathcal{X}$.

An antichain in a causal space $\mathcal{X}$ is a subset of $\mathcal{X}$ whose elements are mutually incomparable. A chain is a totally ordered subset of $\mathcal{X}$.

Given two antichains $\Sigma_{1}, \Sigma_{2}$ in a causal space, we say that $\Sigma_{1} \ll \Sigma_{2}$ if any element of $\Sigma_{1}$ strictly precedes some element of $\Sigma_{2}$, or else is incomparable to all elements of $\Sigma_{2}$, and furthermore, $\Sigma_{1} \cup \Sigma_{2}$ is not an antichain.

Observe that $\ll$ is necessarily anti-reflexive: if $\Sigma \ll \Sigma$ then $\Sigma=\Sigma \cup \Sigma$ is not an antichain (by the definition of $\ll$ ), which is a contradiction.

An immediate predecessor of some element $x \in \mathcal{C}$ is an element $y$ such that $[y, x]=\{x, y\}$. Similarly, an immediate successor is an element $z$ such that $[x, z]=\{x, z\}$.

A causal set $\mathcal{X}$ is said to be connected if it is connected when viewed as an undirected graph. More precisely, given two arbitrary elements $a, b \in \mathcal{X}$, there exists a sequence $x_{0}=a, x_{1}, \cdots, x_{n-1}, x_{n}=b$ such that $x_{i} \prec x_{i+1}$ or $x_{i+1} \prec x_{i}$ for $i=0, \cdots, n-1$.

A foliation of a causal space $\mathcal{X}$ is a partition $\mathcal{F}$ of $\mathcal{X}$ into antichains $\Sigma_{i}, i \in I$ (for $I$ some index set (not necessarily countable!)), such that 
the relation $\ll$ is a strict total order relation on $\mathcal{F}$ ( $\ll$ is transitive, antireflexive $(\forall \Sigma \in \mathcal{F}, \Sigma \ll \Sigma$ does not hold), and any two distinct antichains $\Sigma_{i_{1}}, \Sigma_{i_{2}} \in \mathcal{F}, \Sigma_{i_{1}} \neq \Sigma_{i_{2}}$ are comparable $\left(\Sigma_{i_{1}} \ll \Sigma_{i_{2}}\right.$ or $\left.\Sigma_{i_{2}} \ll \Sigma_{i_{1}}\right)$ ).

Since the relation $\ll$ is anti-reflexive, to see that $\mathcal{F}$ is a foliation it is sufficient to check that $\ll$ is transitive and such that $\left.\ll\right|_{\mathcal{F} \backslash \Delta}$ is a total relation.

A foliation on $\mathcal{X}$ is a temporal foliation if every antichain $\Sigma$ in the foliation satisfies the following condition:

$(\ddagger)$ For any inextendible (or maximal) chain $C \subset \mathcal{X}$, there exist $x, y \in C$ and $z, t \in \Sigma$ such that $x \prec z$ and $t \prec y$.

If $\mathcal{F}$ is a foliation of $\mathcal{X}$, a slice in $\mathcal{F}$ is more succinctly termed $\mathcal{F}$-slice. In case $\Sigma$ is a top slice $\left(\Sigma_{\uparrow}=\Sigma\right)$ then $\Sigma$ satisfies $(\ddagger)$ if and only if $\Sigma \cap C \neq \emptyset$; the same applies in case $\Sigma$ is a bottom slice $\left(\Sigma_{\downarrow}=\Sigma\right)$.

2.2. Causality in Lorentzian manifolds. Let $(\mathcal{M}, \mathrm{g})$ be a spacetime: $\mathcal{M}$ is $n$-differentiable manifold ( $n \geq 2$ ), equipped with a metric $g$ of signature $(-,+\ldots,+)$.

The chronological past of an event $p$ in $\mathcal{M}$, denoted by $I^{-}(p)$, is the set of all events $q \in \mathcal{M}$ such that $p$ can be reached from $q$ using a timelike curve. Similarly, the chronological future of $p, I^{+}(p)$ is the set of events $q$ which can be reached from $p$ using a timelike curve. The causal past/future of $p J^{ \pm}(p)$ is defined analogously by replacing, in the above definition, 'timelike' by 'causal' (where a causal curve is a curve whose tangent vector is nowhere spacelike).

The Alexandrov interval is the open set defined as $I(p, q) \equiv I^{+}(p) \cap I^{-}(q)$. For a globally hyperbolic spacetime $(\mathcal{M}, g)$ we have that $\overline{I(p, q)}=J^{+}(p) \cap$ $J^{-}(q)$ is compact for all $p, q \in \mathcal{M}$.

We denote the relation $q \in I^{-}(p)$ by $q<p$. Similarly, $q \prec p$ is a shorthand for $q \in J^{-}(p)$.

Let $\mathcal{X}$ be a subset of $\mathcal{M}$. Then $\mathcal{X}$ is naturally equipped with a partial order $\prec_{X}=\left.\prec\right|_{\mathcal{X}}$. Henceforth, the order $\prec_{\mathcal{X}}$ will be denoted $\prec$ whenever there is no risk of confusion.

\section{AN AUXILIARY RESULT ON TEMPORAL FOLIATIONS OF CAUSAL SETS}

In this section we show the following:

3.1. Proposition. Let $\mathcal{C}$ be a non-empty causal set. We assume furthermore that $\mathcal{C}$ is countable.

Let $\Sigma_{0}$ be a finite antichain in $\mathcal{C}, \Sigma_{0} \neq \emptyset$. Then there exists an antichain $\Sigma$ containing $\Sigma_{0}$, which satisfies

( ) For any inextendible (or maximal) chain $C \subset \mathcal{C}$, there exist $x, y \in C$ and $z, t \in \Sigma$ such that $x \prec z$ and $t \prec y$. 
Proof. A slice $\Sigma$ satisfying the condition $(\ddagger)$ is called a Cauchy slice. We will construct, inductively (in stages indexed by $i \in \mathbb{N}$ ), antichains $\left\{\Sigma_{i}\right\}_{i}$ satisfying $\Sigma_{i+1} \supseteq \Sigma_{i}$ and a set $\mathcal{X}=\bigcup_{i} \mathcal{X}_{i}$ of maximal chains in $\mathcal{C}$, such that the antichain $\Sigma_{i}$ satisfies $(\ddagger)$ for all chains from $\mathcal{X}_{i}$.

The idea of the proof is to adjoin elements from each chain in $\mathcal{X}$ to $\Sigma_{0}$, if this is possible; the main requirement is that the obtained antichain remains finite at each stage of the procedure; if for some maximal chain $C \in \mathcal{X}$, one cannot add an element of $C$ to the antichain, then necessarily $C$ satisfies $(\ddagger)$.

For any maximal chain $C \subset \mathcal{C}$, define $C_{\prec}$ as the set of elements of $C$ strictly preceding $\Sigma_{0}$, and $C_{\succ}$ be the set of elements strictly succeeding $\Sigma_{0}$.

Before proceeding, let us show that:

$(\dagger)=C_{\prec}$ and $C \neq C_{\succ}$ for any maximal chain $C$.

To see this, note that assuming $C=C_{\prec}$ we obtain a contradiction with the maximality of $C$ : Let $x \in C=C_{\prec}$. For all $z \in \Sigma_{0}$ we have $z \notin C$ (by assumption). By local finiteness of $\mathcal{C}$ the interval $[x, z]$ is finite (or empty) for all such $z$; since $C=C_{\prec}$ by assumption we obtain $C \cap x_{\uparrow} \subset$ $\bigcup_{z \in \Sigma_{0}}[x, z]$, hence finite as $\Sigma_{0}$ is finite; let $x_{0}$ be a maximal element in $C \cap x_{\uparrow}$ (such an element exists as $C \cap x_{\uparrow}$ is finite) and let $z_{0}$ be any element in $\Sigma_{0} \cap x_{0 \uparrow}$, then $C \cup\left\{z_{0}\right\}$ is a chain which strictly contains $C$; this is a contradiction with $C$ being a maximal chain.

The case where $C=C_{\succ}$ is handled similarly.

Now consider the case where $\Sigma_{0 \downarrow}=\mathcal{C}$, (so in particular $\Sigma_{0}$ consists of maximal elements). Here $C=C_{\prec} \cup\left(C \cap \Sigma_{0}\right)$ (any element of $C$ strictly precedes $\Sigma_{0}$ or is in $\Sigma_{0}$ ) for any maximal chain $C$; by the previous argument we obtain $C \cap \Sigma_{0} \neq \emptyset$.

Similarly, if $\Sigma_{0 \uparrow}=\mathcal{C}$ then $C \cap \Sigma_{0} \neq \emptyset$ for any maximal chain $C \subset \mathcal{C}$.

We will assume henceforth that $\Sigma_{0 \downarrow} \neq \mathcal{C}$ and $\Sigma_{0 \uparrow} \neq \mathcal{C}$.

Let us define $\Sigma_{0}^{+}:=\mathcal{C} \backslash \Sigma_{0 \downarrow}$ (then $\Sigma_{0}^{+} \neq \emptyset$ by hypothesis) and $\Sigma_{0}^{-}:=$ $\mathcal{C} \backslash \Sigma_{0}^{+}=\Sigma_{0 \downarrow}$.

Write

$$
\Sigma_{0}^{-}=\left\{x_{1}, x_{2}, \ldots\right\}, \Sigma_{0}^{+}=\left\{y_{1}, y_{2}, \ldots\right\}
$$

and let

$$
\mathcal{Z}=\left\{z_{1}, z_{2}, \cdots\right\}
$$

be an enumeration of $\mathcal{Z}:=\Sigma_{0}^{-} \times \Sigma_{0}^{+}$; elements $z_{i}$ are of the form $z_{i}=$ $\left(x_{k}, y_{\ell}\right), x_{k} \in \Sigma_{0}^{-}, y_{\ell} \in \Sigma_{0}^{+}$(here $(\cdot, \cdot)$ denotes a tuple and not an interval!). The steps of the proof are as follows:

1. For $i=0$ : let $\mathcal{X}_{0}$ be the set of maximal chains $C$ for which $C_{\prec}$ and $C_{\succ}$ are nonempty (in which case $\ddagger$ (with $\Sigma$ replaced by $\Sigma_{0}$ ) holds for $C)$.

2. For $i=1$ : denote by $\mathcal{X}_{1}$ the set of maximal chains in $\mathcal{C}$ containing the elements $x_{m_{1}} \in \Sigma_{0}^{-}, y_{n_{1}} \in \Sigma_{0}^{+}, z_{1}=\left(x_{m_{1}}, y_{n_{1}}\right)\left(m_{1}, n_{1}\right.$ are fixed). 
i. If $y_{n_{1}}$ is incomparable to every element in $\Sigma_{0}$, then we set $\Sigma_{1}=$ $\Sigma_{0} \cup\left\{y_{n_{1}}\right\}$.

ii. Otherwise, any chain containing $x_{m_{1}}, y_{n_{1}}$ will satisfy ( $\left.\ddagger\right)$ since $x_{m_{1}} \prec z \& t \prec y_{n_{1}}$ for some $z, t \in \Sigma_{0}$ by the assumptions on $x_{m_{1}}, y_{n_{1}}$. In this case we set $\Sigma_{1}=\Sigma_{0}$.

3. Assume that we have thus obtained $\mathcal{X}_{i}$ and $\Sigma_{i}$ for all $i \leq k$. Let $i=k+1$ : for any maximal chain $C$, let again $C_{\prec}$ (respectively $C_{\succ}$ ) be the set of elements of $C$ strictly preceding (respectively succeeding) $\Sigma_{k}$.

Denote by $X_{k}$ the set $\Sigma_{0}^{+} \backslash \Sigma_{k \uparrow}$ (so $X_{k}$ is the set of elements incomparable to $\Sigma_{k}$ ).

i. If $X_{k}=\emptyset$ then we set $\Sigma=\Sigma_{k}$. If an element is incomparable to $\Sigma_{k}$ then it is also incomparable to $\Sigma_{0}$ since $\Sigma_{0} \subseteq \Sigma_{k}$; the assumption $X_{k}=\emptyset$ then implies that $\mathcal{C}=\Sigma_{\downarrow} \cup \Sigma_{\uparrow}$. In this case the induction stops at stage $k$ and $\Sigma$ is the sought antichain. For later convenience we also set $\Sigma_{j}=\Sigma$ for all $i \geq k$. Let $C$ be any maximal chain. We have: $C \neq C_{\prec}$ whence $C \cap \Sigma_{\uparrow} \neq \emptyset$; it follows that $(\ddagger)$ holds for $C$ and $\Sigma$.

ii. Otherwise, let $\ell$ be minimal such that $z_{\ell}=\left(x_{m_{\ell}}, y_{n_{\ell}}\right)$ and $y_{n_{\ell}} \in X_{k}$. In this case we set $\Sigma_{k+1}:=\Sigma_{k} \cup\left\{y_{n_{\ell}}\right\}$.

For later reference, observe that $X_{k+1} \subset X_{k} \subset \Sigma_{0}^{+}$, since if an element is incomparable to $\Sigma_{k+1}$ then it is also incomparable to $\Sigma_{k}$ as $\Sigma_{k} \subseteq$ $\Sigma_{k+1}$.

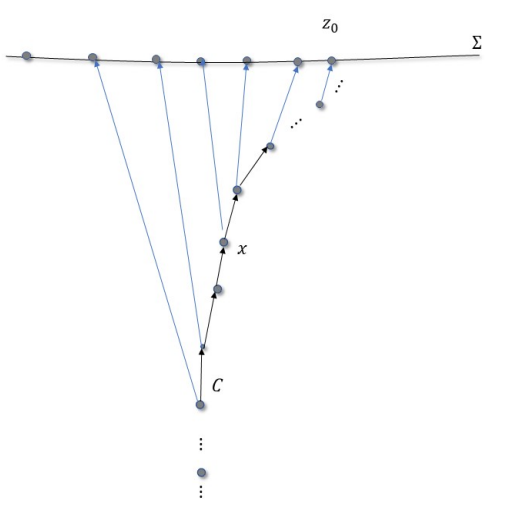

Figure 1. A finite antichain $\Sigma$ and an infinite chain $C$ whose elements lie to the past of $\Sigma$. The causal space $\Sigma \cup C$ (where the order relation is the transitive closure of the arrows shown in the figure) does not satisfy local finiteness and hence is not a causal set. 


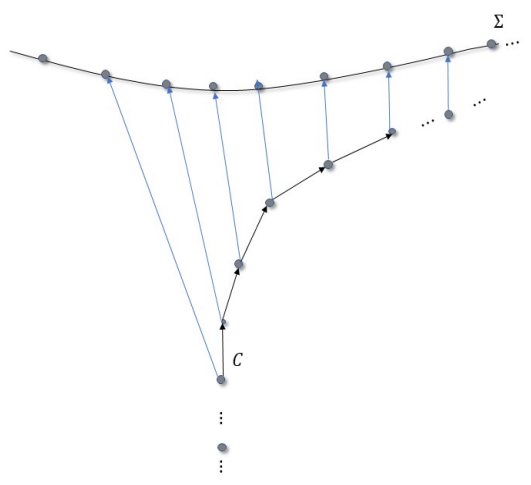

Figure 2. An antichain $\Sigma$ and a maximal chain $C$ whose elements lie to the past of $\Sigma$. Both $\Sigma$ and $C$ are infinite. The causal space $\Sigma \cup C$ satisfies local finiteness, but the requirement $(\ddagger)$ is not met.

We let $\mathcal{X}_{k+1}$ be the set of maximal chains containing the elements $x_{m_{j}}, y_{n_{j}}$ for some $j \in\{1, \cdots, \ell\}$; then by the hypothesis on $x_{m_{j}}, y_{n_{j}}, j=$ $1, \cdots, \ell,(\ddagger)$ holds for all chains in $\mathcal{X}_{k+1}$ and the antichain $\Sigma_{k+1}$.

4. Observe that if $\Sigma \subset \Sigma^{\prime}$ are antichains, with ( $\ddagger$ ) holds for $\Sigma$ and some chain $C$, then necessarily ( $\ddagger)$ holds also for $\Sigma^{\prime}$ and $C$. It follows that in step $k+1$ above, ( $\ddagger)$ holds for the antichain $\Sigma_{k+1}$ and all chains in $\bigcup_{i \leq k+1} \mathcal{X}_{i}$.

5. Let now $\Sigma:=\bigcup_{k \in \mathbb{N}} \Sigma_{k}$, and $\mathcal{X}=\bigcup_{k \in \mathbb{N}} \mathcal{X}_{k}$. Then we show that $(\ddagger)$ holds for $\Sigma$ and all elements in $\mathcal{X}$. Any element (a chain) $C$ of $\mathcal{X}$ will belong to some $\mathcal{X}_{k}$, hence $(\ddagger)$ holds for $C$ and $\Sigma_{k}$ (by the above arguments), thus it will hold for $C$ and $\Sigma$ since $\Sigma \supset \Sigma_{k}$.

6. Note that by construction any element in $\Sigma_{0}^{+}$is now comparable to $\Sigma$, since it will be added to $\Sigma_{k \uparrow}$ at some stage.

7. Note that at each stage $k$, the antichain $\Sigma_{k}$ is finite; hence the reasoning done above (under $(\dagger)$ ) can be repeated with $\Sigma_{0}$ replaced by $\Sigma_{k}$. Let $C$ be a maximal chain; if $C$ intersects $\Sigma_{\downarrow}$ and $\Sigma_{\uparrow}$, there is nothing left to prove. Otherwise, assume ( $\ddagger)$ does not hold for $C$ and $\Sigma$. Since $C$ cannot lie entirely to the past or future of $\Sigma_{k}(\forall k \geq 0)$, $C$ will intersect both $\Sigma_{k \uparrow}$ and $\Sigma_{k \downarrow}$ (for some $k$ ) by the preceding item, hence ( $\ddagger$ ) will be satisfied for $C$ and $\Sigma_{k}$; by the above considerations ( ) will be satisfied for $C$ and $\Sigma$.

We have thus exhausted all possible cases of maximal chains, and $\Sigma$ as defined is a Cauchy slice as required. 


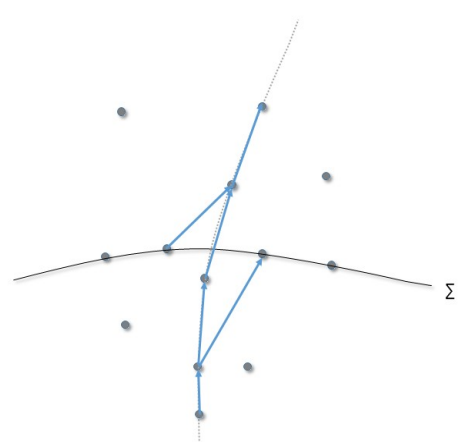

Figure 3. A chain and an antichain satisfying condition ( $\ddagger)$.

3.2. Proposition. Let $\Sigma_{\ell}, \ell \in I \subset \mathbb{Z}, I \neq \emptyset$ be a sequence of disjoint antichains in a given causet $\mathcal{C}$, such that, for no $i \neq j, x \prec y \& z \prec t$ with $x, t \in \Sigma_{i}$ and $y, z \in \Sigma_{j}$.

Then there exists a foliation $\mathcal{F}$ of $\mathcal{C}$ by spacelike slices such that each $\Sigma_{\ell}$ is a subset of some slice of $\mathcal{F}$.

If, furthermore, there are Cauchy slices extending each of the $\Sigma_{\ell}$ 's, then there exists a temporal foliation of $\mathcal{C}$ satisfying the above condition (i.e. each $\Sigma_{\ell}$ is a subset of some slice of $\mathcal{F}$ ).

Proof. The proof will be similar to that of Theorem (2.3) of [3].

We proceed as follows. Let $\mathcal{F}$ be the set of partial foliations $F$ of $\mathcal{C}$, satisfying the following:

(†) For any $\Sigma, \Sigma^{\prime} \in F, \Sigma \neq \Sigma^{\prime}$, if $\Sigma \cap \Sigma_{\ell} \neq \emptyset$ (for some $\ell$ ) then $\Sigma^{\prime} \cap \Sigma_{\ell}=\emptyset$.

Theorem (2.3) of [3] (and its proof, with slight changes) is then just a special case.

The set $\mathcal{F}$ is non-empty (for $\left\{\Sigma_{\ell_{0}}\right\}, \ell_{0} \in I$, is a partial foliation in $\mathcal{F}$ ), and any $\sqsubseteq$-chain in $\mathcal{F}$ is bounded: let $\left(F_{i}\right)_{i}$ be a $\sqsubseteq$-chain in $\mathcal{F}$, then $F_{\text {sup }}$ is the partial foliation composed of unions of $\subset$-chains of slices in $F_{i}$, and $F_{\text {sup }}$ belongs to $\mathcal{F}$. Hence $\mathcal{F}$ admits at least a maximal element. Let $F_{\max }$ be such, then $F_{\max }$ is a total foliation by the proof of Theorem (2.3) of [3].

In case the $\Sigma_{\ell}$ 's can each be extended to a Cauchy slice, then by the end of the aforementioned proof there exists a temporal foliation which satisfies the required properties. 


\section{MAIN RESUlt AND ITS PROOF}

We consider a $C^{\infty}$ Lorentzian $n$-dimensional manifold $\mathcal{M}, n \geq 2$. We assume $\mathcal{M}$ is equipped with a metric $g$ of signature $(-,+, \ldots,+)$ and that $\mathcal{M}$ is time-oriented.

Our goal in this section is to provide a new proof of the following theorem:

4.1. Theorem. Let $\mathcal{M}$ be a globally hyperbolic, geodesically complete spacetime.

Let $\Sigma$ be a $C^{k}$-acausal compact subset of $\mathcal{M}(k \geq 1)$ which is crossed at most once by any inextendible timelike curve. Then there exists a foliation $\mathcal{F}$ of $\mathcal{M}$ by $C^{0}$-acausal hypersurfaces foliating $\mathcal{M}$ such that one of the slices (of the foliation) contains $\Sigma$.

4.2. Outline. We will construct a sequence of open coverings $\left(\mathcal{U}_{k}\right)_{k \in \mathbb{N}}$ of $\mathcal{M}$ which satisfy the following properties:

1. The covering $\mathcal{U}_{k}$ consists of open causal diamonds, i.e. sets of the form $\mathcal{D}(p, q):=I^{+}(p) \cap I^{-}(q)$.

2. For each $k, \mathcal{U}_{k}$ is locally finite, i.e. for every $x \in \mathcal{M}$ there exists a neighbourhood $U \ni x$ which intersects finitely many sets of $\mathcal{U}_{k}$. In particular each $\mathcal{U}_{k}$ is countable.

3. For each $k, \mathcal{U}_{k+1}$ is a refinement of $\mathcal{U}_{k}$, i.e. every element of $\mathcal{U}_{k+1}$ is a subset of some element of $\mathcal{U}_{k}$;

4. Let $\overline{\mathcal{U}}_{k}$ be the family $\left\{\bar{U} \mid U \in \mathcal{U}_{k}\right\}$ (with $\bar{U}$ being the closure of $U$ in the manifold topology). Then the intersection $\bigcap_{k} \bar{U}_{k}$ of any nested sequence $U_{k+1} \subset U_{k}\left(\left(\bar{U}_{k}\right)_{k}, U_{k} \in \mathcal{U}_{k}\right)$, is a singleton.

5. Any point $z \in \mathcal{M}$ lies in some intersection of the above form.

The above can be done in the following way: the manifold $\mathcal{M}$ is covered by the family of open diamonds $\mathcal{D}(p, q), p, q \in \mathcal{M}, p<q$. By paracompactness of $\mathcal{M}$ this cover has a locally finite refinement $\mathcal{U}_{0}^{\prime \prime}$. In order to obtain a locally finite cover by open diamonds, note that any element of $\mathcal{U}_{0}^{\prime \prime}$ has compact closure, hence can be covered by finitely many open diamonds of the above form; for each $U \in \mathcal{U}_{0}^{\prime \prime}$ fix such a cover $\left(\mathcal{D}_{i}\right)_{i \in I_{U}}$; replacing each $U$ by $\left(\mathcal{D}_{i}\right)_{i \in I_{U}}$ we obtain a cover $\mathcal{U}_{0}^{\prime}$; note that $\mathcal{U}_{0}^{\prime}$ is also locally finite.

We can further remove any redundant sets from $\mathcal{U}_{0}^{\prime}$ : discard any set $U \in \mathcal{U}_{0}^{\prime}$ which satisfies $U \subset \bigcup_{i \in I} V_{i}$ for $V_{i} \in \mathcal{U}_{0}^{\prime}, i \in I$ (for some finite $I$ ). This process terminates by local finiteness of $\mathcal{U}_{0}^{\prime}$. The resulting cover, denoted $\mathcal{U}_{0}$, is locally finite as desired.

Similarly, we may consider successive strict refinements of $\mathcal{U}_{0}$ to obtain $\mathcal{U}_{1}, \mathcal{U}_{2}, \cdots$.

In order to ensure that item 4 . above holds we may require that, for each 
$\mathcal{D} \in \mathcal{U}_{k}$,

$$
\delta(\mathcal{D}) \lesssim 2^{-k} \quad \text { in dimensionless units }
$$

where $\delta(\mathcal{D})$ is the maximum timelike separation of any two points in $\overline{\mathcal{D}}$. Now, as $\lim _{k} V\left(\mathcal{D}_{k}\right)_{k}=0$ (for any nested sequence of diamonds $\left(\mathcal{D}_{k}\right)_{k}$ ), the sequence $\left(\overline{\mathcal{D}}_{k}\right)_{k}, \mathcal{D}_{k} \in \mathcal{U}_{k}, \mathcal{D}_{k+1} \subset \mathcal{D}_{k}$ will satisfy $\bigcap_{k} \overline{\mathcal{D}}_{k}=\{z\}$ for some $z \in \mathcal{M}$.

For a 4-dimensional spacetime, the above consideration can be made more transparent by recalling the approximate formula

$$
V(\mathcal{D}(p, q)) \approx \frac{\pi}{24} \tau^{4}
$$

for the spacetime volume of the causal diamond $\mathcal{D}(p, q)$, provided $p, q$ are sufficiently close (and $p \supsetneqq q$ ). In fact in this case there exists a unique timelike geodesic $\gamma(t)$ parametrized by proper time $t \in\left[-\frac{\tau}{2}, \frac{\tau}{2}\right]$ joining $p$ and $q$. Hence the requirement on the timelike separation of $p, q$ is translated to a corresponding requirement on $V(\mathcal{D}(p, q))$. More generally, for an $n$-dimensional spacetime we have (for $p, q$ sufficiently close)

$$
V(\mathcal{D}(p, q)) \approx \operatorname{vol}\left(S^{n-2}\right) \frac{2}{n(n-1)}\left(\frac{\tau}{2}\right)^{n}
$$

where $S^{n-2}$ is the $(n-2)$-sphere.

Finally, for any $z \in \mathcal{M}$ choose, for each $\ell$ some $\mathcal{D}_{\ell} \in \mathcal{U}_{\ell}$ such that $z \in \mathcal{D}_{\ell}$. By the claim above, the intersection of the $\overline{\mathcal{D}}_{\ell}$ 's is a singleton (namely $\{z\})$.

In what follows we will continue to denote $x \in J^{-}(y)$ by $x \prec y$, for any $x, y \in \mathcal{M}$. This applies in particular when considering a discrete set $X \subset \mathcal{M}$ in which case the order on $X$ is the induced causal order.

4.2.1. Main requirements. Using the above construction, we will then obtain an increasing sequence of nested causets $\left(\mathcal{C}_{k}\right)_{k}\left(\mathcal{C}_{k} \subset \mathcal{C}_{k+1}\right)$ and temporal foliations $F_{k}\left(F_{k} \sqsubseteq F_{k+1}\right)$ of $\mathcal{C}_{k}$ such that:

a. For each $k$, an element $U_{i k} \in \mathcal{U}_{k}$ contains $N_{i k}$ elements $x_{i k \ell} \in \mathcal{C}_{k}$, $\ell=1, \ldots, N_{i k}$, where $N_{i k}<\infty$ satisfying some suitable constraints (see below);

b. If $U_{i k} \cap \Sigma \neq \emptyset$ then $x_{i k \ell} \in \Sigma$ for some $\ell$;

c. For any $x_{i k \ell}$ lying to the past or/and future of $\Sigma, x_{i k \ell}$ also lies to the past or/and future of $\Sigma \cap \mathcal{C}_{k}$ (i.e. there exists some $z \in \Sigma \cap \mathcal{C}_{k}$ for which $x_{i k \ell} \prec z$ or/and $z \prec x_{i k \ell}$ respectively);

d. Let $p, q \in \mathcal{C}_{k}, p \neq q$, with $p$ being a direct predecessor of $q$ (i.e. $\nexists x(p \supsetneqq$ $x \supsetneqq q)$ ). Let $\left\{S_{k \ell} \mid \ell=1, \ldots, N\right\}, S_{k 1} \ll \cdots \ll S_{k N}$ be the set of $F_{k^{-}}$ slices $S$ (i.e. $S \in F_{k}$ ) for which $p \supsetneqq z, t \supsetneqq q$ for some $z, t \in S$. Then the set $\overline{\mathcal{D}(p, q)} \cap \mathcal{C}_{k+1}$ contains elements $y_{1} \prec \cdots \prec y_{N} \prec y_{N+1}, z_{\ell}, t_{\ell}$ such that $y_{\ell} \prec z_{\ell}$ and $t_{\ell} \prec y_{\ell+1}$ for some $z_{\ell}, t_{\ell} \in S_{(k+1) \ell}, \ell=1, \cdots, N$ $\left(S_{(k+1) \ell}\right.$ being an $F_{k+1}$-slice). 
More succinctly, the condition $p \prec z, t \prec q$ for some $z, t \in S$ for an $F_{k}$-slice $S$ is termed ' $S$ intersects $\mathcal{D}(p, q)$ '.

Using 4.2.1 as well as the requirements above one can get a rough estimate of the timelike separation between two events $p^{\prime}, q^{\prime} \in \mathcal{D}(p, q)$, $p^{\prime} \in I^{-}\left(q^{\prime}\right)$.

However, for our purposes it is necessary to provide a more precise estimation that holds for all elements of a given causet $\mathcal{C}_{k}$ (for sufficiently high sprinkling density, or equivalently large $k$; here the 'sprinkling' is not completely random since it should always satisfy the above requirements (a through d)); more precisely, we have:

4.3. Assertion. Without loss of generality, the causets $\left\{\mathcal{C}_{k} \mid k \in \mathbb{N}\right\}$ can be constructed so that:

- the timelike separation between two points $p, q, p \in I^{-}(q)$ satisfies

$$
\tau(p, q) \approx \frac{N}{m_{n} \rho^{1 / n} D_{n}^{1 / n}}
$$

(provided $p$ and $q$ are sufficiently close), where $N$ is the length of a maximal chain connecting $p$ to $q, \rho$ is the sprinkling density and $D_{n}, m_{n}$ are constants related to the dimension of $\mathcal{M}$;

- the length of a maximal $\mathcal{C}_{k+1}$-chain connecting $p$ to $q$ (with $p, q \in$ $\left.\mathcal{C}_{k}\right)$ is bounded below by the number of $F_{k}$-slices intersecting $\mathcal{D}(p, q)$.

Proof. The first assertion is equation (3.1) in equation [10] (see also the discussion below Theorem (1.1) (in [1]) reproduced in the appendix). The second assertion follows immediately from requirement (d) of 4.2.1: let $p:=p_{0}, p_{1}, \cdots, p_{s}=: q$ be a maximal chain (of maximal length) joining $p$ to $q$ in $\mathcal{C}_{k}$, then the number of $F_{k}$-slices intersecting $\mathcal{D}(p, q)$ is easily obtained by counting the slices intersecting $\mathcal{D}\left(p_{i}, p_{i+1}\right)$ for $i=$ $0, \cdots, s-1$. By requirement (d), we obtain a new maximal chain by including all the $y_{\ell}$ 's for each interval $\left[p_{i}, p_{i+1}\right]$. For a given interval, the length of the maximal chain (in $\mathcal{C}_{k+1}$ ) joining $p_{i}$ to $p_{i+1}$ is equal to the number of $F_{k}$-slices intersecting $\mathcal{D}\left(p_{i}, p_{i+1}\right)$. The statement then follows.

The above requirements are met by proceeding inductively as follows: start with $k=0$.

4.3.1. Obtaining $\mathcal{C}_{0}$ and $F_{0}$ : Let $\left\{U_{i 0} \mid i \in I_{0} \subseteq \mathbb{N}\right\}$ be an enumeration of the cover $\mathcal{U}_{0}$.

For any $U_{i 0} \in \mathcal{U}_{0}$ we will choose $N_{i 0}$ elements in $U_{i 0}$; these elements will be denoted as $x_{i 0 \ell}\left(\ell=1, \cdots, N_{i 0}\right)$; if $U_{i 0}$ meets $\Sigma$, then some of the $N_{i 0}$ elements in $U_{i 0}$ will belong to $\Sigma$, and other points lie to the future or past of these points.

At this stage, $N_{i 0}$ is just a nonzero natural number (except when $U_{i 0}$ meets $\Sigma$, in such case it must be $\geq 3$ (at least one point is chosen on $\Sigma$, 
and two points in its past and future respectively)).

Set

$$
\mathcal{C}_{0}:=\bigcup_{i \in I_{0}}\left\{x_{i 0 \ell} \mid \ell=1, \cdots, N_{i 0}\right\} .
$$

Since $\mathcal{U}_{0}$ is locally finite, $\mathcal{C}_{0}$ is locally finite (as a causal space): If $p, q, p \supsetneqq$ $q$ are elements of $\mathcal{C}_{0}$, the diamond $\overline{\mathcal{D}(p, q)}$ is compact and is covered by finitely many elements of $\mathcal{U}_{0}$, hence there are only finitely many elements of $\mathcal{C}_{0}$ in the interval $[p, q]:=\overline{\mathcal{D}(p, q)} \cap \mathcal{C}_{0}$, hence the claim.

Let $\Sigma_{0}$ be the set of $x_{i 0 \ell}$ which are in $\Sigma$.

The open sets $\left(U_{i 0}\right)_{i \in I_{0}}$ (for some set $I_{0}$ ) cover $\Sigma$. By compactness of $\Sigma$ we may extract a finite subcover $\left\{U_{j 0} \mid j \in J\right\}$. As the cover $\mathcal{U}_{0}$ is locally finite, the family of open $U_{i 0}$ having nonempty intersection with $\Sigma$ is finite, hence $\Sigma_{0}$ is finite.

By (the proof of) proposition 3.1 it is possible to extend $\Sigma_{0}$ to a Cauchy slice $\Sigma_{0}^{\prime}$ of $\mathcal{C}_{0}$. Let again $I_{0}^{\prime}$ be the set of indices of open sets $U_{i 0} \in \mathcal{U}_{0}$ meeting $\Sigma_{0}^{\prime}$, and let $X_{0}:=\left(\mathcal{C}_{0} \cap \bigcup_{i \in I_{0}^{\prime}} U_{i 0}\right) \backslash \Sigma_{0}^{\prime}$.

Let also $X_{0}^{+}=X_{0} \cap\left(\Sigma_{0}^{\prime} \cup \Sigma\right)_{\uparrow}$ and $X_{0}^{-}=X_{0} \cap\left(\Sigma_{0}^{\prime} \cup \Sigma\right)_{\downarrow}$. Fix some $a \in X_{0}^{-}$(since $X_{0}^{-}$is nonempty (by construction)). Then $\{a\}$ is an antichain. Apply Proposition 3.2 to $X_{0}^{-}$(with $\{a\}$ playing the role of the $\Sigma_{\ell}$ 's) to obtain a partition of $X_{0}^{-}$into slices $\Gamma_{0-\ell}$. Similarly, apply 3.2 to $X_{0}^{+}$(since $X_{0}^{+}$is nonempty by construction) to obtain a partition of $X_{0}^{+}$ into Cauchy slices $\Gamma_{0+\ell}$. Apply proposition 3.2 again (with the antichains

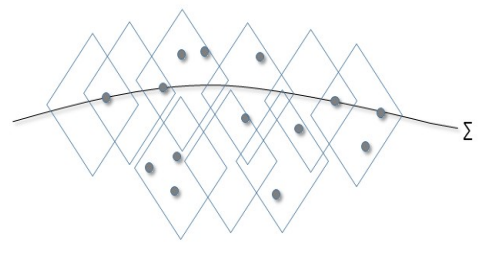

Figure 4. Points 'sprinkled' on $\Sigma$ and in its past and future developments.

$\Sigma_{0}^{\prime}, \Gamma_{0-\ell}, \Gamma_{0+\ell}$ 's playing the role of $\Sigma_{\ell}$ 's) to get a temporal foliation $F_{0}$ of $\mathcal{C}_{0}$ such that $\Sigma_{0}^{\prime}$ is a subset of some slice of $F_{0}$. 
It can be seen that items $(\mathrm{a}, \mathrm{b}, \mathrm{c})$ above are met for $(k=0)$ (item $\mathrm{d}$ concerns only $k \geq 1$ ).

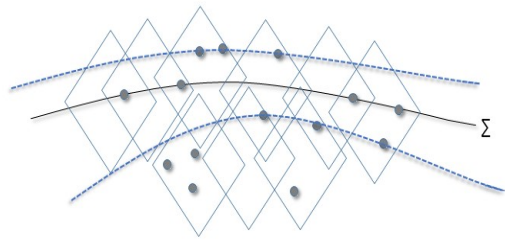

Figure 5. The procedure described above allows to ensure that the antichain $\Sigma_{0}^{\prime}$ and its extensions in $F_{k}$ do not have extraneous points (i.e. points past or future to $\Sigma$.)

4.3.2. Extending $\Sigma_{0}^{\prime}$ to a Cauchy slice. A crucial step is to verify that a Cauchy slice in $F_{0}$ (in particular $\Sigma_{0}^{\prime}$ ) remains extendible to a Cauchy slice after adding countably many elements to $\mathcal{C}_{0}$ :

4.4. Lemma. Keep the above notation. Let $\mathcal{C}_{0}^{\prime}$ be a connected causal set extending $\mathcal{C}_{0}\left(\mathcal{C}_{0} \subseteq \mathcal{C}_{0}^{\prime}\right.$ and the order induced from $\mathcal{C}_{0}^{\prime}$ coincides with $\left.\prec\right)$. Then there exists a Cauchy slice $\Sigma_{0}^{\prime \prime} \subset \mathcal{C}_{0}^{\prime}$ extending $\Sigma_{0}^{\prime}$.

Proof. Observe that since $\Sigma_{0}^{\prime}$ is a Cauchy slice then $J\left(\Sigma_{0}^{\prime}\right):=J^{+}\left(\Sigma_{0}^{\prime}\right) \cup$ $J^{-}\left(\Sigma_{0}^{\prime}\right)$ will meet any open set $U \in \mathcal{U}_{0}$ by the properties of $\mathcal{U}_{0}$.

Assume that ( $\ddagger$ ) (from section 3 ) does not hold for $\Sigma_{0}^{\prime}$ and $C^{\prime}$, where $C^{\prime} \subset \mathcal{C}_{0}^{\prime}$ is some maximal chain. Then either $J^{-}\left(C^{\prime}\right):=\bigcup_{x \in C^{\prime}} J^{-}(x)$ or $J^{+}\left(C^{\prime}\right):=\bigcup_{x \in C^{\prime}} J^{+}(x)$ does not intersect $\Sigma_{0}^{\prime}$. Observe that $C^{\prime}$ is infinite (otherwise $C^{\prime}$ would cease to be inextendible). Then $J^{ \pm}\left(C^{\prime}\right)$ will consist of connected non compact subsets of $\mathcal{M}$. Hence either $J^{-}\left(\Sigma_{0}^{\prime}\right)$ or $J^{+}\left(\Sigma_{0}^{\prime}\right)$ does not meet a connected non-compact subset of $\mathcal{M}$. Since $\Sigma_{0}^{\prime}$ is a Cauchy slice in $\mathcal{C}_{0}^{\prime}$, we obtain a contradiction with the assumptions concerning the cover $\mathcal{U}_{0}$.

4.4.1. Obtaining $\mathcal{C}_{k}$ and $F_{k}$ for $k \geq 1$. Assume that we have obtained causets $\mathcal{C}_{\ell}$ and temporal foliations $F_{\ell}$ for $\ell=0,1, \cdots, k$. By replacing $\mathcal{C}_{\ell}$ with $\bigcup_{i<\ell} \mathcal{C}_{i}$, we may assume that $\mathcal{C}_{\ell} \subset \mathcal{C}_{\ell+1}$ for $\ell=0, \cdots, k-1$. We will Repeat the procedure done in 4.3 .1 with some changes. 
Let $\left\{U_{i(k+1)} \mid i \in I_{k+1} \subseteq \mathbb{N}\right\}$ be an enumeration of the cover $\mathcal{U}_{k+1}$. For any $U_{i(k+1)} \in \mathcal{U}_{k+1}$ choose $N_{i(k+1)}$ elements (denoted as $x_{i(k+1) \ell}$ with $\left.\ell=1, \cdots, N_{i(k+1)}\right)$ in $U_{i(k+1)}$; this is done subject to the requirement in item (d) above: more precisely, if $p$ is an immediate predecessor to $q$, $p, q \in \mathcal{C}_{k} \cap U_{i(k+1)}$, let $\left\{S_{k \ell} \mid \ell=1, \ldots, N\right\}, S_{k 1} \ll \cdots \ll S_{k N}$ be the set of $F_{k}$-slices $S$ for which $p \prec z, t \prec q$ for some $z, t \in S$. Then the elements $x_{i(k+1) \ell}$ contain distinct elements $y_{2}, \cdots, y_{N}, z_{m}, t_{m}$ with $m=1, \cdots, N$ such that $\left(z_{m} \| t_{m}\right.$ or $\left.z_{m}=t_{m}\right)$ for $m=1, \cdots, N$ and

$$
p=: y_{1} \prec \cdots \prec y_{N+1}:=q \&\left(y_{m} \prec z_{m} \& t_{m} \prec y_{m+1}\right) \text { for } m=1, \cdots, N \text {. }
$$

Set

$$
\mathcal{C}_{k+1}:=\bigcup_{i \in I_{k+1}}\left\{x_{i(k+1) \ell} \mid \ell=1, \cdots, N_{i(k+1)}\right\} \cup \mathcal{C}_{k} .
$$

Let $\Sigma_{k+1}$ be the set $\mathcal{C}_{k+1} \cap \Sigma$. The same procedure done in 4.3.1 is then repeated for $\Sigma_{k+1}$, provided appropriate changes are made. We deduce that $\Sigma_{k+1}$ is finite.

Define $\Sigma_{k+1}^{\prime}:=\Sigma_{k+1} \cup \Sigma_{k}^{\prime}$ (where $\Sigma_{k}^{\prime}$ has already been obtained at step $k$ ). Let again $I_{k+1}^{\prime}$ be the set of indices of opens $U_{i(k+1)} \in \mathcal{U}_{k+1}$ meeting $\Sigma_{k+1}^{\prime}$, and let $X_{k+1}:=\left(\mathcal{C}_{k+1} \cap \bigcup_{i \in I_{k+1}^{\prime}} U_{i(k+1)}\right) \backslash \Sigma_{k+1}^{\prime}$. Let also $X_{k+1}^{+}=$ $X_{k+1} \cap\left(\Sigma_{k+1}^{\prime} \cup \Sigma\right)_{\uparrow}$ and $X_{k+1}^{-}=X_{k+1} \cap\left(\Sigma_{k+1}^{\prime} \cup \Sigma\right)_{\downarrow}$. Let $\left\{\Gamma_{i(k+1)}^{-} \mid i \in I^{-}\right\}$ (with $I^{-} \subset \mathbb{Z}$ ) be the set of $F_{k}$-slices contained in $X_{k+1}^{-}$augmented with all elements $y_{\ell}$ from item (d) above; more precisely, $\Gamma_{i(k+1)}^{-}$are given by

$$
\Gamma_{i(k+1)}^{-}=S \cup \bigcup_{\left\{p, q \in \mathcal{C}_{k+1} \mid\{p\} \ll^{\prime} S \ll^{\prime}\{q\}\right\}}\left\{z, t \in \mathcal{C}_{k+1} \backslash \mathcal{C}_{k} \mid p \prec^{\prime} z \& t \prec^{\prime} q\right\}
$$

where $S$ is an $F_{k}$-slice contained in $X_{k+1}^{-}, \ll^{\prime}$ denotes immediate precedence among antichains in $\mathcal{C}_{k+1}$, and $\prec^{\prime}$ is the immediate causal precedence in $\mathcal{C}_{k+1}$. Apply proposition 3.2 to $X_{k+1}^{-}$with the slices $\Gamma_{i(k+1)}^{-}$ playing the role of the $\Sigma_{\ell}$ 's to obtain a partition of $X_{k+1}^{-}$into Cauchy slices $\Gamma_{0-\ell}$.

A similar procedure is applied to $X_{k+1}^{+}$to obtain a partition (of $X_{k+1}^{+}$) into slices $\Gamma_{0+\ell}$. Now let $\left\{\Gamma_{i(k+1)} \mid i \in I\right\}$ (with $I \subset \mathbb{Z}, I \neq \emptyset$ ) be the set of $F_{k}$-slices augmented with all elements $y_{\ell}$ from item (d) above; more precisely, $\Gamma_{i(k+1)}$ are given by

$$
\Gamma_{i(k+1)}=S \cup \bigcup_{\left\{p, q \in \mathcal{C}_{k+1} \mid\{p\} \ll^{\prime} S \ll^{\prime}\{q\}\right\}}\left\{z, t \in \mathcal{C}_{k+1} \backslash \mathcal{C}_{k} \mid p \prec^{\prime} z \& t \prec^{\prime} q\right\}
$$

where $S$ is an $F_{k^{-}}$-slice, $\ll^{\prime}$ and $\prec^{\prime}$ are as above.

Apply proposition 3.2 to $\mathcal{C}_{k+1}$ with the antichains $\left\{\Gamma_{i(k+1)}, \Gamma_{0-\ell}, \Gamma_{0+\ell} \mid i \in\right.$ $I$ ) playing the role of $\Sigma_{\ell}$ 's to get a temporal foliation $F_{k+1}$ with $F_{k} \sqsubseteq$ $F_{k+1}$. Here $\sqsubseteq$ designates the following relation among foliations: $F \sqsubseteq F^{\prime}$ iff every slice of $F$ is a subset of a slice of $F^{\prime}$.

It can now be seen that items (a, b, c, and d) above are met for all $k \geq 1$. 


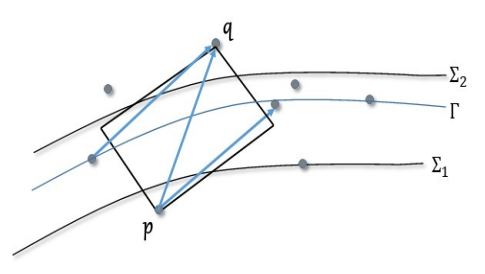

Figure 6 . Two points $p, q$ of $\mathcal{C}_{k}$ and a $F_{k}$-slice $\Gamma$ satisfying conditions in (c) of 4.2.1.

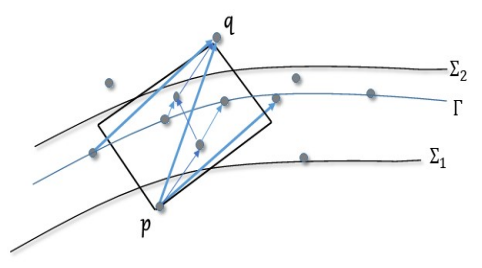

Figure 7 . The added points to $\mathcal{C}_{k+1}$ along the lines of requirements in item (d) in 4.2.1.

We have:

4.5. Assertion. Given any $p, q \in \mathcal{C}_{k}, p \supsetneqq q$ for some $k \in \mathbb{N}$, there exists some $\ell \geq k$ and $a z \in \mathcal{C}_{\ell}$ such that $p \supsetneqq z \supsetneqq q$.

Proof. Let $\mathcal{V}_{0}$ be the spacetime volume of the diamond $\mathcal{D}(p, q)$; by the conditions imposed on the covers $\left(\mathcal{U}_{\ell}\right)_{\ell}$, we see that for some sufficiently large $\ell, \mathcal{D}(p, q)$ is covered by a minimum of at least three diamonds 
$U_{0}, U_{1}, U_{2} \in \mathcal{U}_{\ell}$, with $p \in U_{0} \backslash U_{1}, q \in U_{2} \backslash U_{1}$; then clearly there is a $z \in U_{1} \cap \mathcal{C}_{\ell}$ for which $p \supsetneqq z \supsetneqq q$.

Given a slice $S \in F_{\ell}$, (in particular $S \neq \emptyset$ ), let $k_{0} \leq \ell$ be the smallest $k \in \mathbb{N}$ such that there is $S^{\prime} \in F_{k}\left(S^{\prime} \neq \emptyset\right.$ as $F_{k}$ is a foliation) satisfying $S^{\prime} \subset S$. We set $k(S):=k_{0}$ thus defined; in particular, for $S \in F_{\ell}, S^{\prime} \in$ $F_{\ell^{\prime}}, \ell, \ell \in \mathbb{N}, k(S)=k\left(S^{\prime}\right)$ whenever $S^{\prime} \subset S$ or $S \subset S^{\prime}$.

Define the set $\mathcal{F}^{\dagger}$ of sequences of slices in $\bigcup_{\ell} F_{\ell}$ :

$$
\begin{aligned}
\left(S_{k}\right)_{k \geq k_{0}} \in \mathcal{F}^{\dagger} \quad \longleftrightarrow \quad \exists N \in \mathbb{N}, k_{0}:=k\left(S_{N}\right) \\
\text { and } \forall k \in \mathbb{N}, \quad k \geq k_{0}, S_{k} \in F_{k} \& S_{k+1} \supseteq S_{k} .
\end{aligned}
$$

Let $\mathcal{C}:=\bigcup_{k} \mathcal{C}_{k}$ be the union of the causets $\mathcal{C}_{k}$. By 4.5 the space $\mathcal{C}$ is a causal space, and not a causal set, since it does not satisfy the requirement of local finiteness.

Let $\mathcal{F}$ be the following partition of $\mathcal{C}$ : The elements of $\mathcal{F}$ are sets of the form

$$
S\left(\left(S_{k}\right)_{k \geq k_{0}}\right)=\left(\bigcup_{k \geq k_{0}} S_{k}\right)
$$

with $\left(S_{k}\right)_{k \geq k_{0}} \in \mathcal{F}^{\dagger}$.

We will show first that $\mathcal{F}$ is a foliation of $\mathcal{C}$. For this purpose we will show that the following axioms hold for $\mathcal{F}$ :

(1) The foliation $\mathcal{F}$ is a cover of $\mathcal{C}$ : let $z \in \mathcal{C}$ be an arbitrary element, then $z \in \mathcal{C}_{k}$ for some $k$ (by definition of $\mathcal{C}$ ), then $z \in S_{k} \in F_{k}$ (since $F_{k}$ is a cover of $\mathcal{C}_{k}$ ), whence $z \in S$ with $S=S\left(\left(S_{\ell}^{\prime}\right)_{\ell}\right)$ and $\left(S_{\ell}^{\prime}\right)_{\ell} \in \mathcal{F}^{\dagger}$ is the sequence satisfying $S_{k}^{\prime}=S_{k}$.

(2) Any two distinct slices of $\mathcal{F}$ are disjoint: this follows since otherwise we would have some $z \in S_{k} \cap S_{\ell}^{\prime}$, for $S_{k} \in F_{k}, S_{\ell}^{\prime} \in F_{\ell}$ (and $S_{k} \nsubseteq S_{\ell}^{\prime}, S_{\ell}^{\prime} \nsubseteq S_{k}$ ). Hence (assuming $k>\ell$, say) $z \in S_{k} \cap S_{k}^{\prime}$, is a contradiction with $F_{k}$ being a foliation.

(3) For $S, S^{\prime} \in \mathcal{F}, S \ll S^{\prime}$ or $S^{\prime} \ll S$ (with « being the order relation on slices induced from $\prec$ in an obvious way $\left(S \ll S^{\prime}\right.$ iff $\left.\exists z \in S \exists z^{\prime} \in S^{\prime}\left(z \prec z^{\prime}\right)\right)$ : this can be seen immediately by recalling the definitions of $S, S^{\prime}$ as unions of slices in $\mathcal{C}_{k}$ 's, and using the axioms of a foliation.

Observe that $\mathcal{F}$ equipped with the order $\ll$ is a countable dense total order without endpoints. Then by standard order theory $\mathcal{F}$ is order isomorphic to $\mathbb{Q}$.

The Dedekind completion of $\mathcal{F}$ with respect to the order $\ll$ is order isomorphic to $\mathbb{R}$. We will denote it by $\widehat{\mathcal{F}}$. We have $\mathcal{F} \subset \widehat{\mathcal{F}}$.

Let $S \in \widehat{\mathcal{F}}$. We declare that $z \rightarrow S$ if $z=\lim _{k} z_{k}$ with $z_{k} \in S_{k}$ (with $S_{k} \in \mathcal{F}$ ) and $S=\lim _{k} S_{k}$ (in the «-order topology (generated by intervals) on $\mathcal{F}$ ) for all but finitely many $k$ 's. 
4.5.1. 'Completing' the foliation. Let

$$
\mathcal{F}^{\prime}:=\{\{z \in \mathcal{M} \mid z \rightarrow S\} \mid S \in \widehat{\mathcal{F}}\} .
$$

Observe that each $S \in \mathcal{F}$ is a subset of some $S^{\prime} \in \mathcal{F}^{\prime}$, since $z \in S$ implies $z \rightarrow S$ by considering the constant sequence $z_{k}=z$. However, we do not have $\mathcal{F} \subset \mathcal{F}^{\prime}$ in general, since a slice in $\mathcal{F}$ needs to be somehow completed in order to get a slice of $\mathcal{F}^{\prime}$.

Consider the following map

$$
\iota: \widehat{\mathcal{F}} \rightarrow \mathcal{F}^{\prime}, S \mapsto S^{\prime}:=\{z \in \mathcal{M} \mid z \rightarrow S\},
$$

then $\iota$ is a bijection. Surjectivity follows by construction. Let us show that $\iota$ is injective: assume otherwise, so we have $\iota(S)=\iota\left(S^{\prime}\right)$ for some $S, S^{\prime} \in \widehat{\mathcal{F}}, S \neq S^{\prime}$; in particular $S=\inf _{k} S_{k} \ll S^{\prime}=\sup _{\ell} S_{\ell}^{\prime}$ for $\left(S_{k}\right)_{k}$ (respectively $\left(S_{\ell}^{\prime}\right)_{\ell}$ ) a sequence of $\mathcal{F}$-slices.

For sufficiently large $k, \ell$ there is a finite spacetime gap (i.e. the causal diamond between any two causally related points of $S_{k}, S_{\ell}$ has a spacetime volume larger than a minimal strictly positive quantity ) and hence we cannot have $(\forall z \in \iota(S)) z \rightarrow S \& z \rightarrow S^{\prime}$.

We have:

4.6. Proposition. The set $\mathcal{F}^{\prime}$ covers $\mathcal{M}$. Furthermore, if $S \ll S^{\prime}$ for distinct $S, S^{\prime} \in \widehat{\mathcal{F}}$ then for all $z \in \iota(S), \nexists z^{\prime} \in \iota\left(S^{\prime}\right)\left(z^{\prime} \supsetneqq z\right)$.

Proof. Let $p \in \mathcal{M}$ be arbitrary; there exists a sequence $\left(p_{k}\right)_{k}, z_{k} \in \mathcal{C}$ converging to $p$; hence the set $X:=\left\{p_{k} \mid k \in \mathbb{N}\right\}$ is bounded and contained in a compact subset of $\mathcal{M}$. It follows that $X$ meets any causet $\mathcal{C}_{\ell}$ in a finite set. We can then extract a subsequence $\left(p_{\ell}^{\prime}\right)_{\ell} \equiv\left(p_{k_{\ell}}\right)_{\ell}$ which converges to $p$ with $p_{\ell}^{\prime} \in T_{\ell} \in F_{\ell}$. Hence $p \rightarrow T, T:=\lim _{\ell} T_{\ell}$ and $p \in \iota(T)$.

To show the second assertion assume that for some $z \in \iota(S)$ there exists a $z^{\prime} \in \iota\left(S^{\prime}\right)$ such that $z^{\prime} \supsetneqq z$.

Observe that $\mathcal{D}\left(z^{\prime}, z\right) \cap \mathcal{C} \neq \emptyset$ has no isolated points; in particular $z=$ $\lim _{k} z_{k}=\sup _{k}\left\{z_{k}\right\}, z^{\prime}=\lim _{\ell} z_{\ell}^{\prime}=\inf _{\ell}\left\{z_{\ell}^{\prime}\right\}($ as $\mathcal{C}$ is dense in $\mathcal{M})$ with $z_{k}, z_{\ell}^{\prime} \in \mathcal{C} \cap \mathcal{D}\left(z^{\prime}, z\right)$ for all $k, \ell$.

Then for large enough $k, \ell z_{\ell}^{\prime} \prec z_{k}$. Let, for all $k, \ell, S_{k}, S_{\ell}^{\prime} \in \mathcal{F}$ such that $z_{k} \in S_{k}, z_{\ell}^{\prime} \in S_{\ell}$. Necessarily $S_{\ell}^{\prime} \ll S_{k}$ (by the properties of $\mathcal{F}$ ), hence $S^{\prime} \ll S$, contradicting the antisymmetry of $\ll$.

Equip $\mathcal{F}^{\prime}$ with the induced order, also denoted by $\ll\left(\iota\left(S_{1}\right) \ll \iota\left(S_{2}\right)\right.$ iff $S_{1} \ll S_{2}$ ).

\subsubsection{The slices of $\mathcal{F}^{\prime}$ are Cauchy hypersurfaces.}

4.7. Proposition. Each slice of $\mathcal{F}^{\prime}$ is crossed only once by any inextendible timelike curve.

Proof. The statement can be split into two parts: 
i. The intersection of each inextendible timelike curve with any $\mathcal{F}^{\prime}$-slice is non-empty, and

ii. Any timelike curve cannot intersect an $\mathcal{F}^{\prime}$-slice in more than one point.

Let us show statement (i) first: In order to show the required result, we will assume the contrary.

( $\star \star)$ Assume that there exist an inextendible timelike curve $C$ and a slice $S \in \mathcal{F}^{\prime}$, such that $C \cap S=\emptyset$.

We may assume (without loss of generality) that $C$ can be approximated by a maximal chain in $\mathcal{C}_{k}$ (at each stage $k$ ).

In order to satisfy this assumption, we will modify slightly the family of causets $\left(\mathcal{C}_{k}\right)_{k}$ constructed above. More precisely, consider the covering $\mathcal{U}_{k}$ and let $\left(\mathcal{C}_{k}^{\prime}\right)_{k}$ be a family of causets dependent on $C$ that, in addition to items (a) through (d), satisfy the following condition

$(*)$ The set $C_{k}:=\left\{p \mid p \in U \cap \mathcal{C}_{k}^{\prime}\right.$, for $U \in \mathcal{U}_{k}$ and $\left.U \cap C \neq \varnothing\right\}$, is totally ordered.

Having constructed the sequence of causets $\mathcal{C}_{k}^{\prime}$, the same considerations and related constructions for the causets $\mathcal{C}_{k}$ are repeated in the present context. For simplicity, we will continue to denote $\mathcal{F}$ and $\mathcal{F}^{\prime}$ the corresponding foliations of the causal space $\mathcal{C}^{\prime}=\bigcup_{k} \mathcal{C}_{k}^{\prime}$ and $\mathcal{M}$ respectively.

There are two cases:

(1) $S \in \iota(\mathcal{F})$, i.e. $S=\iota\left(S\left(\left(S_{k}\right)_{k}\right)\right)$ for some $\left(S_{k}\right)_{k} \in \mathcal{F}^{\dagger}$, (2) $S \in \mathcal{F}^{\prime} \backslash \mathcal{F}$.

In case (1): The causets $\mathcal{C}_{k}^{\prime}$ can be chosen in such a way that $C_{k}$ is a total order (this is possible starting from any given $p_{k 0} \in U \cap \mathcal{C}_{k}$ for $U \in \mathcal{U}_{k} U \cap C \neq \varnothing$, by choosing inductively subsequent points in the causal pasts or future of previous points in $C_{k}$ ). The foliations $F_{k}$ can in turn be chosen so that $S_{k} \in F_{k}$ (which is possible by proposition 3.2). Since each $F_{k}$ is a temporal foliation of $\mathcal{C}_{k}$, we have:

$$
\exists x, y \in C_{k} \exists z, t \in S_{k} \text { such that } x \prec z \& t \prec y
$$

We let $x_{k}$, (respectively $y_{k}$ ) be a maximal (respectively minimal) element in $C_{k}$ satisfying the above condition.

It follows from the above considerations that for some $x, y \in C, z, t \in S$, $x \prec z \& t \prec y$.

We obtain: by clause (d) above (under 4.2.1), the maximality of $C_{k}$ in $\mathcal{C}_{k}$ and the choice of $x_{k}, y_{k}$, it follows that $x_{k}, y_{k} \rightarrow p$ for some unique $p$ (as $k \rightarrow \infty$ ) and hence $p \in C \cap S$ as required. More precisely, at each stage k, the sets $C_{k} \cap \mathcal{D}\left(x_{k_{0}}, y_{k_{0}}\right)$ (for some sufficiently large integer $k_{0}$ ) become more and more dense; hence the timelike distance between $x_{k}, y_{k}$ tends to zero as $k$ grows indefinitely. 
In case (2): $S$ is the limit of slices $S_{\ell}^{\prime} \in \mathcal{F}, \ell \in \mathbb{N}$ (in the topology associated to the order $\ll)$. By case (1), the curve $C$ intersects each $S_{\ell}^{\prime}$ once: let $z_{\ell}^{\prime}$ be the intersection point $\left\{z_{\ell}^{\prime}\right\}=C \cap S_{\ell}^{\prime}$. Then $z:=\lim _{\ell} z_{\ell}^{\prime}$ exists and belongs to $C \cap S$ is the unique intersection point of $C$ with $S$. In particular one has $C \cap S \neq \emptyset$.

It follows from the above reasoning that the intersection of $C$ and $S$ is nonempty, contrary to the hypothesis; the obtained contradiction then proves the desired claim.

The proof of statement (ii) proceeds exactly through the same steps, provided that one replaces $C \cap S=\emptyset$ in $(\star \star)$ by $\#(C \cap S)>1$ and then deriving a contradiction.

We have the following:

4.8. Proposition. The family $\mathcal{F}^{\prime}$ is a foliation of $\mathcal{M}$.

Proof. We have already shown that $(4.6) \mathcal{F}^{\prime}$ is a covering of $\mathcal{M}$.

Any two distinct elements of $\mathcal{F}^{\prime}$ are disjoint. To see this, assume otherwise, and let $z \in S \cap S^{\prime}, S, S^{\prime} \in \mathcal{F}^{\prime}, S \ll S^{\prime}, S \neq S^{\prime}$.

Let $C$ be an inextendible timelike curve, passing through $z$. The existence of such a curve follows for instance by considering a small causal diamond containing $z$ and its image by a causal homeomorphism into $\mathbb{M}^{n}(n$ dimensional Minkowski spacetime). The curve $C$ crosses $S, S^{\prime}$ at $z$. Since it intersects all slices $S^{\prime \prime}$ which satisfy $S \ll S^{\prime \prime} \ll S^{\prime}$ (by 4.7 ) then $z \in S^{\prime \prime}$ (otherwise there exists some $z^{\prime \prime} \in S^{\prime \prime} \cap C$ for which $z \supsetneqq z^{\prime \prime}$ or $z^{\prime \prime} \supsetneqq z$ contradicting $S \ll S^{\prime \prime} \ll S^{\prime}$ by 4.6 ). It follows that for infinitely many slices $\iota\left(S_{\ell}\right), S_{\ell} \in \mathcal{F}$ satisfying $S \ll \iota\left(S_{\ell}\right) \ll S^{\prime}$ one has $z \in \iota\left(S_{\ell}\right)$.

Let us see how this contradicts the construction of $\mathcal{C}_{k}$ and $F_{k}$ for $k \in \mathbb{N}$.

As in the proof of 4.7 above, we approximate $C$ by sets $C_{k}$ in $\mathcal{C}_{k}$, and we may assume (without loss of generality) that $C_{k}$ is a chain for all $k$. Let $p, q$ be close points on $C, p \supsetneqq q$, and let $S_{1}, S_{2}$ be two $\mathcal{F}$-slices such that $p \prec t \& u \prec q$ for some $t \in S_{1}, u \in S_{2}$. It can be seen that when $k$ increases, $C_{k}$ approaches $C$ and the elements $p_{k}, q_{k}$ approaching $p, q$ respectively belong to $C_{k}$.

Using the assumption on the timelike separation in $\mathcal{C}_{k}$ (4.3.1) we have (with $N$ being the number of $F_{k}$ slices intersecting $\mathcal{D}(p, q)$ and lying between $S_{1}$ and $S_{2}$ ):

$$
\tau\left(p_{1}, p_{2}\right) \geqq \frac{N}{m_{n} \rho^{1 / n} D_{n}^{1 / n}}
$$

for $p_{1} \in C_{k} \cap S_{1}, p_{2} \in C_{k} \cap S_{2}$; by the assumption $z \in S_{1} \cap S_{2}$, it follows that $\tau\left(p_{1}, p_{2}\right) \rightarrow 0$ for $p_{1}, p_{2} \rightarrow z$ as $k \rightarrow \infty$. However, the quantity $\frac{N}{m_{n} \rho^{1 / n} D_{n}^{1 / n}} \nrightarrow 0($ as $k \rightarrow \infty)$ since otherwise $S_{1}=S_{2}$, thus obtaining a contradiction. In particular this shows that the intersection of $C$ with the union of slices $S^{\prime \prime}, S \ll S^{\prime \prime} \ll S^{\prime}$ cannot be a singleton. 
Let now $S, S^{\prime} \in \mathcal{F}^{\prime}$ be two distinct slices; as was already shown in Proposition 4.6, if $S \ll S^{\prime}$ then there cannot exist an element $z^{\prime} \in S^{\prime}$ which precedes an element $z \in S$.

It remains to show that every $S \in \mathcal{F}^{\prime}$ is an antichain (with respect to the order $\prec$ ). Let $z, z^{\prime} \in S$, such that $z \neq z^{\prime}$. Then we show that we cannot have $z \prec z^{\prime}$ or $z^{\prime} \prec z$. If this were the case, say $z \prec z^{\prime}$, then using $z=\lim _{\ell \rightarrow \infty} z_{\ell}$ and $z^{\prime}=\lim _{k \rightarrow \infty} z_{k}^{\prime}$ (with $z_{k}, z_{\ell}^{\prime} \in \mathcal{C} \cap \mathcal{D}\left(z^{\prime}, z\right)$ for all $k, \ell$ ) we obtain $z_{\ell}^{\prime} \prec z_{k}$ for large enough $k, \ell$. Similarly to the above, we obtain $S_{\ell}^{\prime} \ll S_{k}$ for large enough $k, \ell\left(\right.$ say $k, \ell>N$ ), where $z_{\ell}^{\prime} \in S_{\ell}^{\prime}, z_{k} \in S_{k}$. Now let $S_{m} \in \mathcal{F}, S_{\ell}^{\prime} \ll S_{m} \ll S_{k}, S_{m}$ distinct than $S_{k}$, $S_{\ell}^{\prime}$, for all $k, \ell>N$. By the properties of Dedekind completion we get $S \ll \iota\left(S_{m}\right) \ll S$, obtaining a contradiction with the antisymmetry of $\ll$.

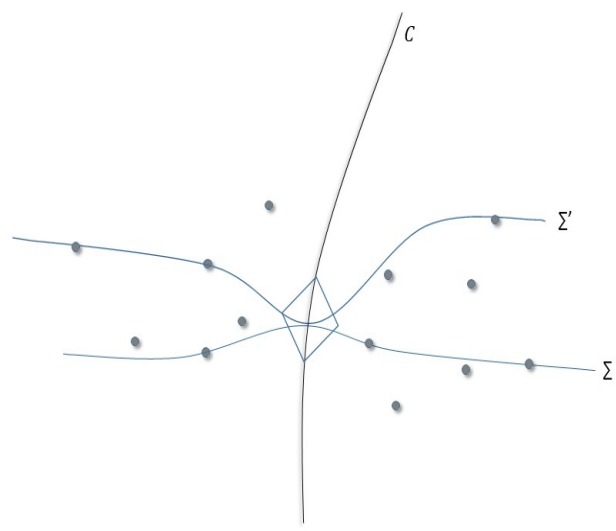

Figure 8. The slices in the figure have an unwanted feature: they intersect at a point.

The rest of the proof is probably standard facts on causal spaces (in particular it does not build on causal set theory).

4.9. Proposition. Each slice $S \in \mathcal{F}^{\prime}$ is a topological manifold of dimension $n-1$.

Proof. We will equip each $S$ with the induced topology from $\mathcal{M}$. So we have to show that every open subset of $S$ is in fact homeomorphic to (an open subset of) $\mathbb{R}^{n-1}$. Let $V \subset S$ be open, then $V=U \cap S$ for some open $U \subset \mathcal{M}$. We may assume that $U$ is a bounded subset, i.e. $U$ is contained in some chronological diamond $\mathcal{D}:=I^{-}(q) \cap I^{+}(p), p \neq q \in \mathcal{M}$.

Let $\phi: U \rightarrow U_{n} \subset \mathbb{R}^{n}$ be a (local) causal isomorphism, where $U_{n}$ is some open subset of $\mathbb{M}^{n}$. 
We have to show that there exists a homeomorphism $\psi: V \rightarrow V_{n-1}$ with $V_{n-1}$ an open in $\mathbb{R}^{n-1}$.

Observe that each timelike curve passing through $U$ intersects $V$ at most once (otherwise two distinct elements of $V$ would be comparable).

We may assume furthermore that the map $\phi$ can be extended to $\overline{\mathcal{D}}$, where $\mathcal{D}:=I^{-}(q) \cap I^{+}(p), p \neq q \in \mathcal{M}$ (as above) is an open causal diamond containing $U$.

Let $\mathcal{S}^{\prime}$ be a partition of $\phi(\mathcal{D})$ into timelike curves joining $\phi(p)$ to $\phi(q)$. The inverse image of each timelike curve in $\phi(\mathcal{D})$ will be a timelike curve in $\mathcal{D}$; in particular $\mathcal{S}:=\phi^{-1}\left(\mathcal{S}^{\prime}\right):=\left\{\phi^{-1}(C) \mid C \in \mathcal{S}^{\prime}\right\}$ is a partition of $\mathcal{D}$ into (disjoint) timelike curves.

Let $\Sigma_{1}$ be any Cauchy hypersurface crossing $\phi(\mathcal{D})$ transversally, i.e. any timelike curve joining $\phi(p)$ to $\phi(q)$ inside $\phi(\mathcal{D})$ will cross $\Sigma_{1}$ only once; this is clearly possible.

There exists a homeomorphism $\psi_{1}: \Sigma_{1} \cap \phi(U) \rightarrow V_{n-1}$, where $V_{n-1}$ is an open subset of $\mathbb{R}^{n-1}$.

Consider the map $\psi$ defined as follows:

Given any $m \in S \cap U$, let $C_{m}$ be the timelike curve in $\mathcal{S}$ which passes through $m$; then $\psi$ sends $m$ to the image $\psi_{1}\left(\phi\left(C_{m}\right) \cap \Sigma_{1}\right)$. It can be seen that $\psi$ is a bijection.

Now let $V^{\prime}$ be another open $\subset S$ having non-empty intersection with $V$, and let $\phi^{\prime}$ be a corresponding bijection $\phi^{\prime}: V^{\prime} \rightarrow V_{n-1}^{\prime}$ (with obvious notation). Furthermore, it is trivial to see (by standard properties of continuous maps) that

$$
\left.\phi^{\prime} \circ \phi^{-1}\right|_{\phi\left(V \cap V^{\prime}\right)}: \phi\left(V \cap V^{\prime}\right) \rightarrow \phi^{\prime}\left(V \cap V^{\prime}\right),
$$

(with $\phi\left(V \cap V^{\prime}\right) \subset V_{n-1}$ and $\left.\phi^{\prime}\left(V \cap V^{\prime}\right) \subset V_{n-1}^{\prime}\right)$ is a homeomorphism. Our procedure then shows that $S$ is a topological manifold of dimension $n-1$ as required.

Combining the above elements and renaming $\mathcal{F}^{\prime}$ as $\mathcal{F}$ we conclude the proof of Theorem 4.1 .

\section{EXISTENCE OF TIME FUNCTIONS}

In this section we consider the issue of the existence of global time functions on a globally hyperbolic manifold.

5.1. Theorem. Let $\mathcal{M}$ be a globally hyperbolic, geodesically complete spacetime. Let $\Sigma_{0}$ be a $C^{0}$ achronal compact subset of $\mathcal{M}$, which is crossed at most once by any inextendible timelike curve. Then there exists a continuous, strictly increasing time function $t: \mathcal{M} \rightarrow \mathbb{R}$ such that $t^{-1}(0) \supseteq \Sigma_{0}$ and for each $r \in \mathbb{R}, t^{-1}(r)$ is a Cauchy hypersurface.

Proof. Recall that $\mathcal{F}$, equipped with the induced order $\ll$, is a countable dense linear order without endpoints. It is known that any such order 
is isomorphic to $(\mathbb{Q}, \leq)$ (with $\leq$ the standard order on the rationals). Hence there is a (non-canonical) isomorphism $\iota: \mathcal{F} \rightarrow \mathbb{Q}$ which satisfies furthermore $\iota\left(\Sigma_{0}^{\prime}\right)=0$, where $\Sigma_{0}^{\prime} \supseteq \Sigma_{0}$ is the $\mathcal{F}$-slice containing $\Sigma_{0}$.

As $\widehat{\mathcal{F}}$ is the Dedekind completion of $\mathcal{F}$, the map $\iota$ has a unique extension to a map $f: \widehat{\mathcal{F}} \rightarrow \mathbb{R}$ satisfying $f\left(\Sigma_{0}^{\prime}\right)=0$.

We denote by $\widehat{\Sigma}_{0}^{\prime}$ the $\mathcal{F}^{\prime}$-slice containing $\Sigma_{0}^{\prime}$. Using the order isomorphism $i: \mathcal{F}^{\prime} \rightarrow \widehat{\mathcal{F}}$ (see the proof of Theorem 4.1), we obtain a new map $f^{\prime}: \mathcal{F}^{\prime} \rightarrow \mathbb{R}, f^{\prime}=f \circ i$. Since $f\left(\Sigma_{0}^{\prime}\right)=0$ it follows that $f^{\prime}\left(\widehat{\Sigma}_{0}^{\prime}\right)=0$.

Now define $\left(\Sigma\right.$ being an arbitrary element of $\left.\mathcal{F}^{\prime}\right)$

$$
t: \mathcal{M} \rightarrow \mathbb{R}, t(z)=f^{\prime}(\Sigma) \text { with } z \in \Sigma .
$$

It can now be seen that the map $t$ satisfies the conditions:

- $t$ is strictly increasing and continuous: this follows from its construction,

- $t^{-1}(0) \supset \Sigma_{0}$, and

- for all $r \in \mathbb{R}, \Sigma:=t^{-1}(r)$ is a Cauchy hypersurface, as required.

\section{The SLICES ARE ALMOST EVERYWHERE SPACELIKE}

In this section we will show that the temporal splitting obtained in Theorem 4.1 consists of slices possessing spacelike tangent spaces defined almost everywhere. To achieve this we make use of the following version of Rademacher's Theorem (see, e.g. [7] corrolary 1.5):

6.1. Proposition. Let $Z$ be a measurable subset of the Euclidean space $\mathbb{R}^{n}$ and let $f$ be a locally Lipschitz map to $\mathbb{R}$, then for almost all $z \in Z$ the differential $D_{z} f$ exists and the restriction $D_{z} f: \mathbb{R}^{n} \rightarrow D_{z} f\left(\mathbb{R}^{n}\right)$ is linear.

We obtain:

6.2. Theorem. Let $\mathcal{M}$ be a globally hyperbolic, geodesically complete spacetime.

Let $\Sigma$ be a $C^{k}$-acausal compact subset of $\mathcal{M}(k \geq 1)$ which is crossed at most once by any inextendible timelike curve. Then there exists a foliation $\mathcal{F}$ of $\mathcal{M}$ by $C^{0}$-acausal hypersurfaces foliating $\mathcal{M}$ such that one of the slices (of the foliation) contains $\Sigma$. Furthermore, the slices are almost everywhere spacelike, i.e. for any $\Sigma^{\prime} \in \mathcal{F}$, there exists a null measurable subset $N \subset \Sigma^{\prime}$ such that for all $p \in \Sigma^{\prime} \backslash N, T_{p} \Sigma^{\prime}$ exists and is spacelike.

Proof. By the proofs of Theorems 4.1 and 5.1 we obtain a foliation $\mathcal{F}$ and a time function $t: \mathcal{M} \rightarrow \mathbb{R}$, constant on $\mathcal{F}$-slices.

Let $\Sigma^{\prime} \in \mathcal{F}$ be some slice. We have $t\left(\Sigma^{\prime}\right)=t_{0}=$ const. Let $U$ be some open set in $\mathcal{M}$ intersecting $\Sigma^{\prime}$. 
Let $\phi: U \rightarrow U_{n} \subset \mathbb{M}^{n}$ be a causal isomorphism; then for $p \in U$, $\phi(p)=\left(x^{\mu}(p)\right)_{\mu=0, \cdots, n-1}$. Let $V \subset \mathbb{R}^{n-1}$ be the projection of $\phi\left(U \cap \Sigma^{\prime}\right)$ on the last $n-1$-coordinates.

Define the following map: $X^{0}: V \rightarrow \mathbb{R}$, by the requirement:

$$
t\left(\phi^{-1}\left(X^{0}\left(x^{1}, \cdots, x^{n-1}\right), x^{1}, \cdots, x^{n-1}\right)\right)=t_{0} .
$$

We will show first that $X^{0}$ is locally Lipshitz; for any $p \in \Sigma^{\prime} \cap U$ there exists an open causal diamond $\mathcal{D} \subset U$ containing $p$ such that: for any $p^{\prime}, q^{\prime} \in \mathcal{D} \cap \Sigma^{\prime},\left|x^{0}\left(p^{\prime}\right)-x^{0}\left(q^{\prime}\right)\right|<C \cdot \max \left\{\left|x^{i}\left(p^{\prime}\right)-x^{i}\left(q^{\prime}\right)\right|, i=1, \cdots, n-1\right\}$ (for some constant $C>0$ ) since $p^{\prime}$ and $q^{\prime}$ are incomparable. It follows that

$$
\left|X^{0}\left(x^{i}\left(p^{\prime}\right)\right)-X^{0}\left(x^{i}\left(q^{\prime}\right)\right)\right|<C \cdot \max \left\{\left|x^{i}\left(p^{\prime}\right)-x^{i}\left(q^{\prime}\right)\right|, i=1, \cdots, n-1\right\} .
$$

The last inequality holds for all $p^{\prime}, q^{\prime} \in \mathcal{D} \cap \Sigma^{\prime}$, hence it holds for a sufficiently small open set in $V$ (contained in the projection of $\phi\left(\mathcal{D} \cap \Sigma^{\prime}\right)$ ) on the last $n-1$ coordinates).

Since $p$ was arbitrary, we see that $X^{0}$ is locally Lipshitz as claimed.

By Rademacher's Theorem, the map $X^{0}$ then possess directional derivatives (along each $x^{i}$, for $i=1, \ldots, n-1$ ) for almost all $\left(x^{i}\right)_{i=1, \cdots, n-1} \in V$. These directional derivatives allow us to obtain a spacelike tangent space at $p$ to $\Sigma^{\prime}$ for all $p \in U \cap \Sigma^{\prime} \backslash N$ with $N \subset \Sigma^{\prime}$ null measurable. Since $U$, $\Sigma^{\prime}$ and $\mathcal{D}$ were arbitrary, the Theorem follows.

\section{Appendix A. Relation Between timelike Distance And LENGTH OF MAXIMAL CHAIN}

Recall the following result (Theorem (1.1) [1]):

A.1. Theorem. Let $\mathcal{D}$ be a compact spacetime domain with volume $\mathcal{V}=$ 1. Let $a, b \in \mathcal{D}$ be such that $a \prec b$.

Let $P$ denote a discrete spacetime associated with $\mathcal{D}$, then, for any $\varepsilon>0$, w.h.p.

$$
\left|\frac{L_{P}(a, b)-c_{d} L_{D}(a, b) n^{1 / d}}{n^{1 / d}}\right|<\varepsilon
$$

where $c_{d}$ is a constant which depends only on the dimension. Moreover, given any Riemannian metric which is compatible with the differential structure of $\mathcal{M}$, and any $\varepsilon>0$ the maximal chain in $P$ between $a$ and $b$ will be contained w.h.p. in an $\varepsilon$-neighbourhood of a maximal curve in $\mathcal{D}$ between $a$ and $b$.

Here $n$ denotes the cardinality of $P$ and an event is said to occur with high probability (w.h.p.) whenever its probability approaches 1 as $n$ tends towards infinity; $L_{P}$ denotes the length of a maximal sized chain joining $a$ to $b$ and $L_{D}:=\sup _{C} L(C)$ where $C$ ranges over all timelike curves joining $a$ to $b$ and contained in $D$.

From the above one can deduce the following: 
a. For any $\delta, \varepsilon, 0<\delta<1, \varepsilon>0$, there exists some integer $N>0$ such that for all $n>N$ the probability of the event $\left|\frac{L_{P}(a, b)-c_{d} L_{D}(a, b) n^{1 / d}}{n^{1 / d}}\right| \geq \varepsilon$ is less than $\delta$.

b. In the notation of section 4, (but assuming that the sprinkling process is a Poisson process, in particular a random process) given a sequence of covers $\left(\mathcal{U}_{k}\right)_{k}$, each $\mathcal{U}_{k}$ consisting of open diamonds $U_{i k}$, let $U_{k_{0}} \in \mathcal{U}_{k_{0}}$ be a fixed open diamond. Then the probability of the event $\left|\frac{L_{\mathcal{C}_{k}}(a, b)-c_{d} L_{\bar{U}_{i k}}(a, b) n^{1 / d}}{n^{1 / d}}\right| \geq \varepsilon$ for all $a, b \in \bar{U}_{i k} \cap \mathcal{C}_{k}$ and for all, $k \geq k_{0}$ with $U_{i k} \subset U_{k_{0}}$; with $n$ being the cardinality of $\mathcal{C}_{k} \cap \bar{U}_{i k}$, is zero.

It follows from these observations that there is no loss of generality of assuming that

$$
\frac{L_{\mathcal{C}_{k}}(a, b)}{n^{1 / d}} \simeq c_{d} L_{\bar{U}_{i k}}(a, b),
$$

simply by avoiding a set of zero measure in the appropriate probability space.

\section{REFERENCES}

1. Bachmat E 2008 "Discrete spacetime and its applications", Contemp. Math. 458 347-59 (arXiv:gr-qc/0702140).

2. Bombelli, L. \& Meyer, D., "The origin of Lorentzian geometry", Phys. Lett. A, Volume 141, number 5,6, (1989).

3. Bleybel, A. \& Zaiour, A. Found Phys (2018) 48: 456.; doi.org/10.1007/s10701018-0157-0.

4. Antonio N. Bernal and Miguel Sánchez, "On smooth Cauchy hypersurfaces and Geroch's splitting theorem", Com. in Math. Phys. 243 (2003), no. 3, 461-470.

5. Antonio N. Bernal and Miguel Sánchez, "Further results on the smoothability of Cauchy hypersurfaces and Cauchy time functions", Lett. Math. Phys. 77 (2006) 183-197.

6. Geroch, R., Domain of dependence, J. Math. Phys. 11, 437 (1970); doi.org/10.1063/1.1665157.

7. A. Lytchak, "Differentiation in metric spaces", St. Petersburg Math. J. Tom. 16 (2004), vyp. 6 Vol. 16 (2005), No. 6, Pages 1017-1041.

8. Martin, K. \& Panangaden, P. Commun. Math. Phys. (2006) 267: 563. https://doi.org/10.1007/s00220-006-0066-5.

9. Seth Major, David Rideout, and Sumati Surya, "On recovering continuum topology from a causal set", J. Math. Phys. 48, 032501 (2007).

10. Rideout, D \& Wallden, P 2009, 'Spacelike distance from discrete causal order', Classical and quantum gravity, vol. 26, no. 15. https://doi.org/doi:10.1088/0264$9381 / 26 / 15 / 155013$.

Faculty of Sciences (I), Lebanese University, Beirut, Lebanon 
Figures

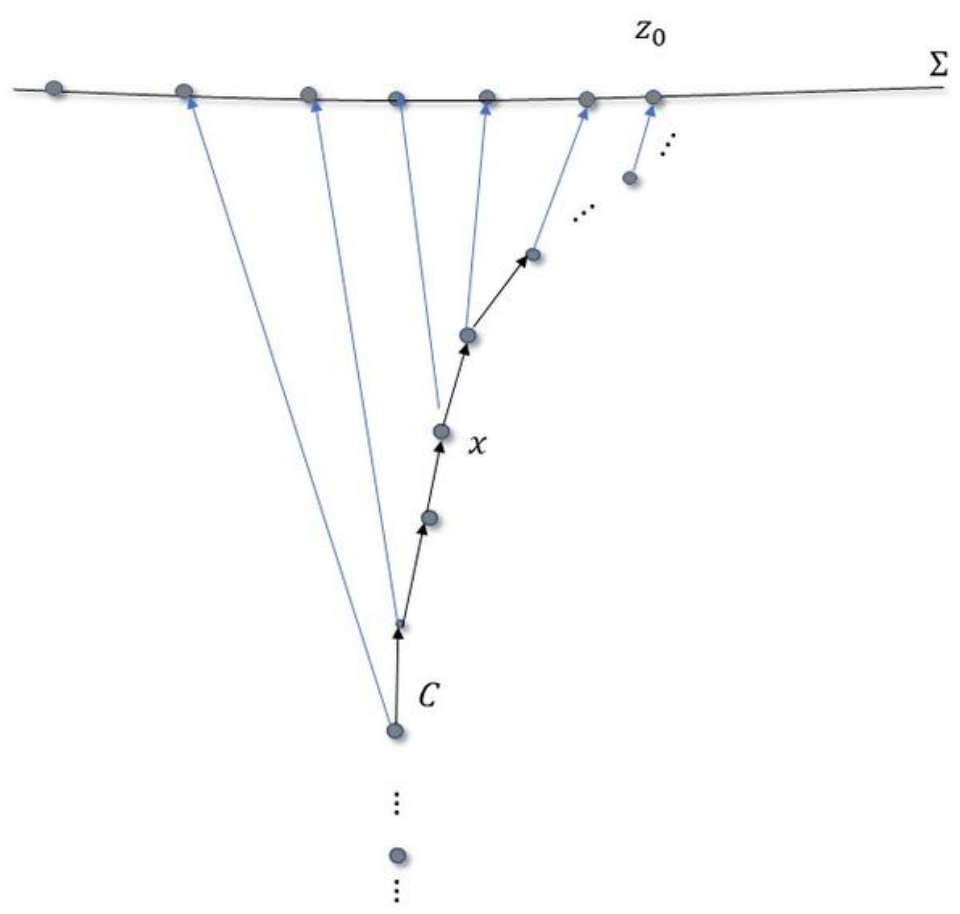

\section{Figure 1}

A nite antichain $\Sigma$ and an innite chain $C$ whose elements lie to the past of $\Sigma$. The causal space $\Sigma \nabla C$ (where the order relation is the transitive closure of the arrows shown in the figure) does not satisfy local finiteness and hence is not a causal set. 


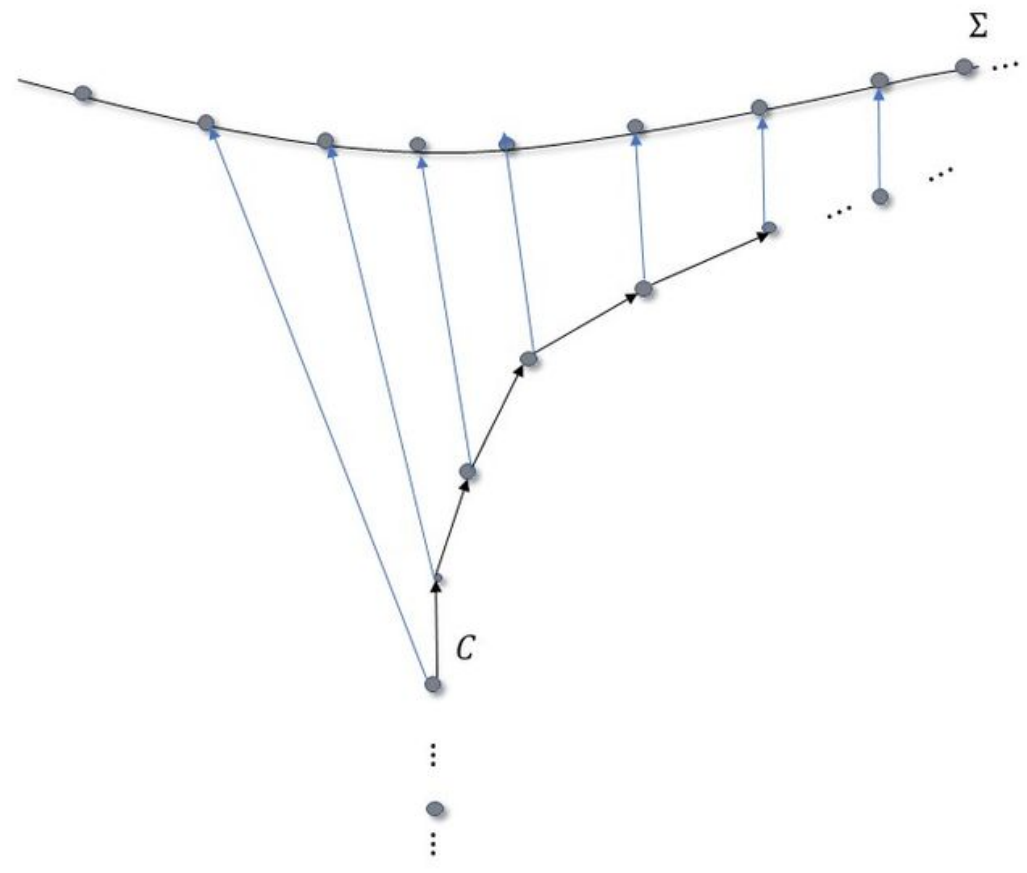

\section{Figure 2}

An antichain $\Sigma$ and a maximal chain $C$ whose elements lie to the past of $\Sigma$. Both $\Sigma$ and $C$ are infinite. The causal space $\Sigma \llbracket C$ satises local finiteness, but the requirement $(\ddagger)$ is not met.

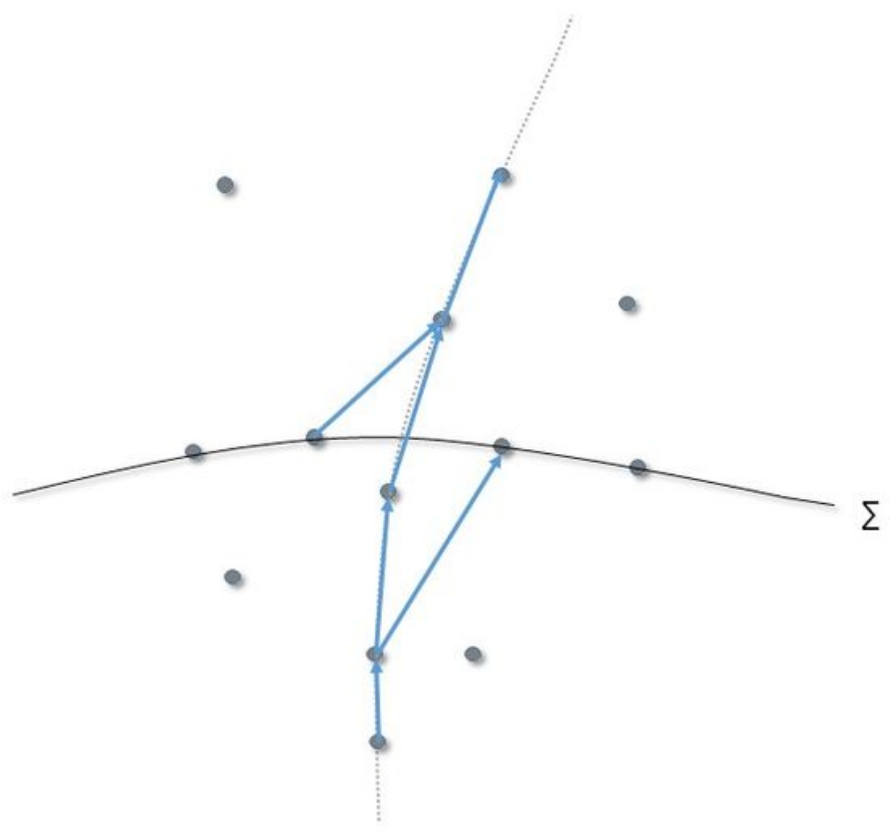


Figure 3

A chain and an antichain satisfying condition ( $¥)$.

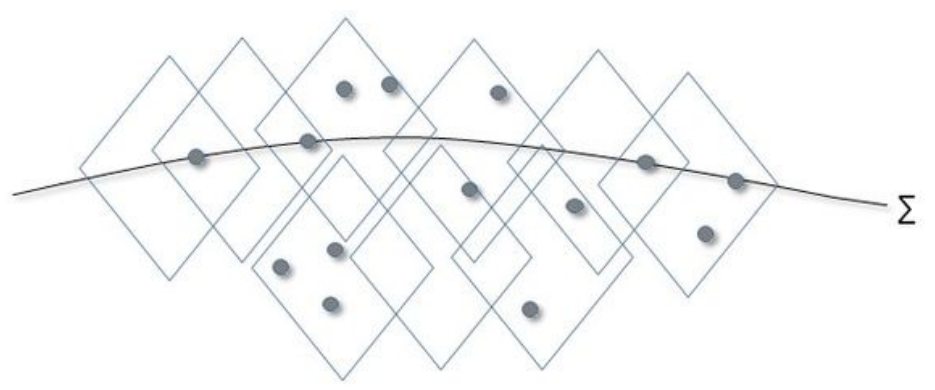

\section{Figure 4}

Points 'sprinkled' on $\Sigma$ and in its past and future developments. 


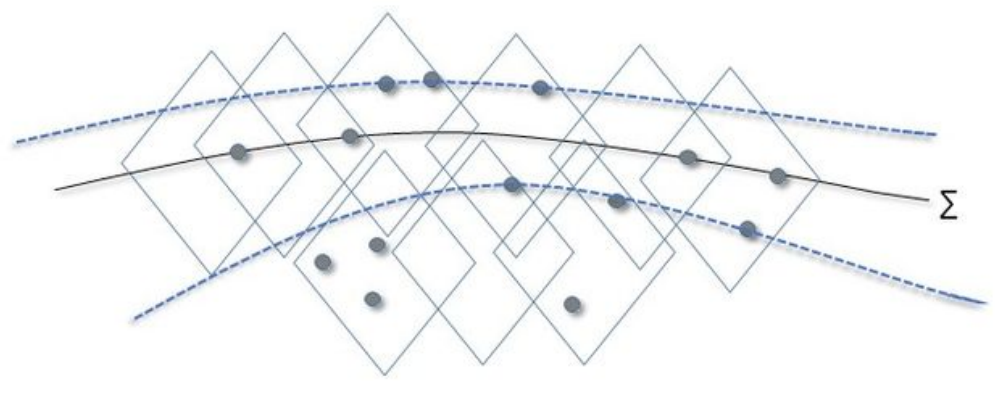

Figure 5

The procedure described above allows to ensure that the antichain $\Sigma^{\prime} 0$ and its extensions in Fk do not have extraneous points (i.e. points past or future to $\Sigma$.)

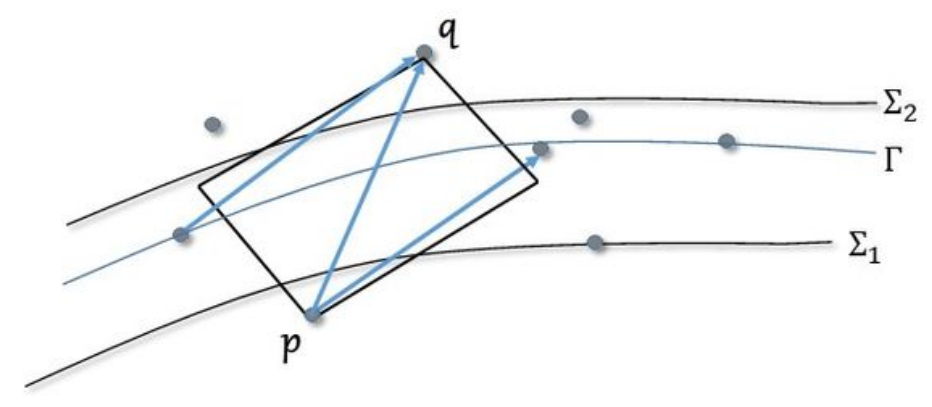


Figure 6

Two points p, q of Ck and a Fk-slice $\Gamma$ satisfying conditions in (c) of 4.2.1.

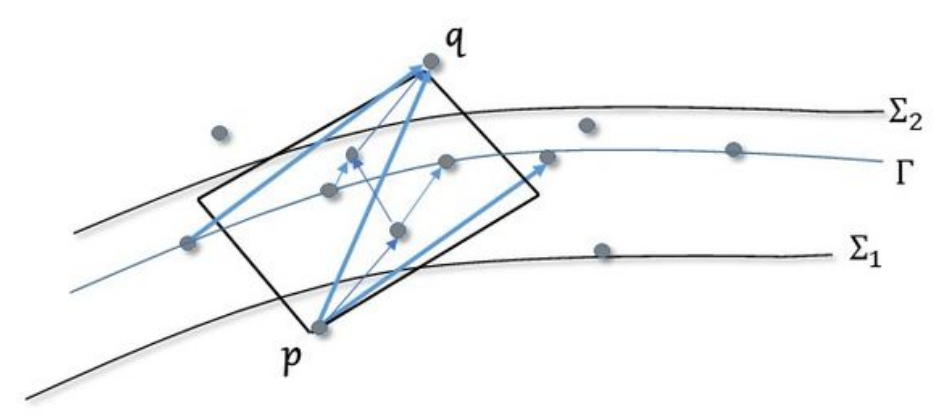

\section{Figure 7}

The added points to $\mathrm{Ck}+1$ along the lines of requirements in item (d) in 4.2.1. 


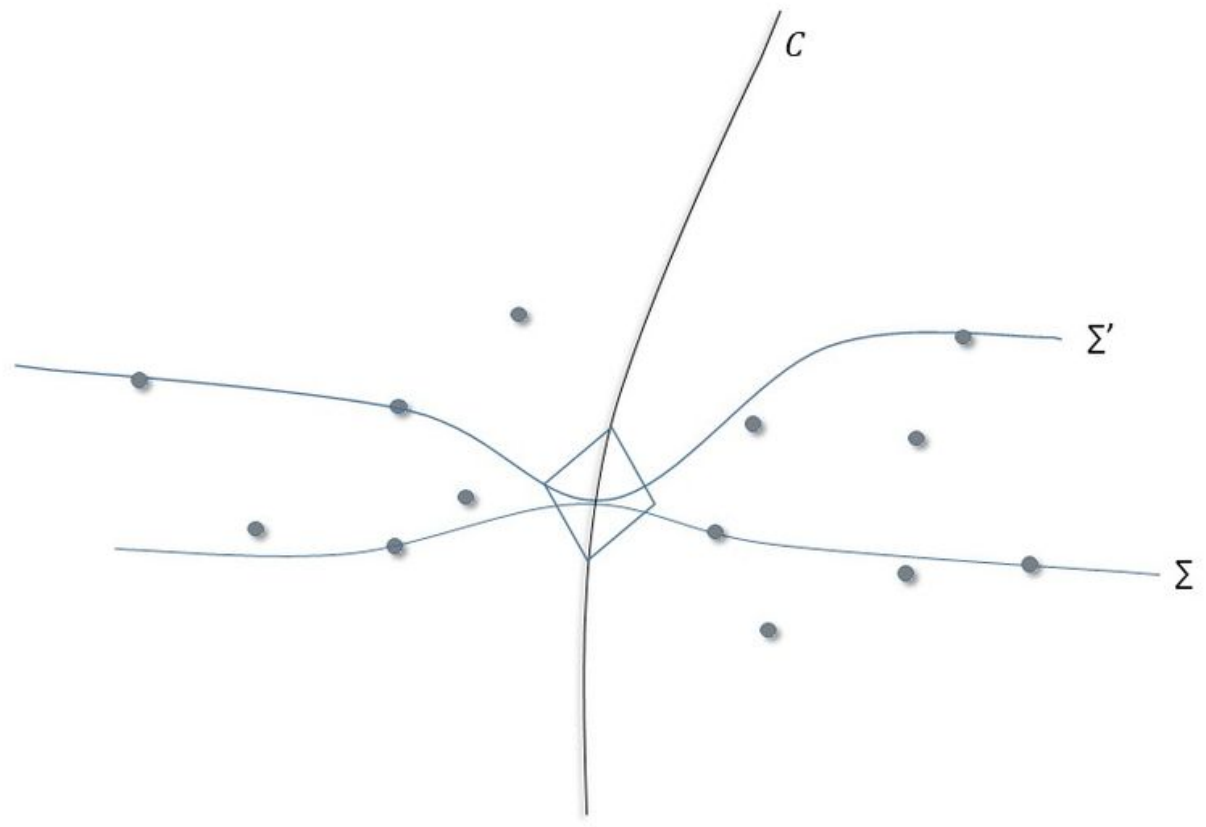

Figure 8

The slices in the figure have an unwanted feature: they intersect at a point. 